\title{
RANDOM WEIGHTED SOBOLEV INEQUALITIES AND APPLICATION TO QUANTUM ERGODICITY
}

\author{
by
}

Didier Robert \& Laurent Thomann

\begin{abstract}
This paper is a continuation of [18 where we studied a randomisation method based on the Laplacian with harmonic potential. Here we extend our previous results to the case of any polynomial and confining potential $V$ on $\mathbb{R}^{d}$. We construct measures, under concentration type assumptions, on the support of which we prove optimal weighted Sobolev estimates on $\mathbb{R}^{d}$. This construction relies on accurate estimates on the spectral function in a non-compact configuration space. Then we prove random quantum ergodicity results without specific assumption on the classical dynamics. Finally, we prove that almost all basis of Hermite functions is quantum uniquely ergodic.
\end{abstract}

\section{Introduction and results}

1.1. Introduction. - The aim of this paper is to prove stochastic properties of weighted Sobolev spaces associated to the Schrödinger operator

$$
\hat{H}=\hat{H}_{V}=-\Delta+V,
$$

in $L^{2}\left(\mathbb{R}^{d}\right)$ where $V$ is a real confining polynomial potential. We will see that most of the results obtained in $1 \mathbf{1 8}$ for the harmonic oscillator $\left(V(x)=|x|^{2}\right)$ have a natural extension in this context. In the case of the harmonic oscillator we can take profit of many explicit formulas and both the spectrum and the eigenfunctions are well understood. This is not true anymore for more general potentials even if many asymptotic properties are known (see $[\underline{4}, \mathbf{1 0}, \mathbf{1 5}, \mathbf{1 9}]$ ). The results obtained in this paper combine accurate spectral results for the operator $\hat{H}_{V}$ with probabilistic methods. Our work is inspired by similar properties proved before by Burq and Lebeau [2] (see also Shiffman and

2000 Mathematics Subject Classification. - 35R60; 35P05 ; 35J10 ; 58J51.

Key words and phrases. — Schrödinger operator with confining potential, spectral analysis, quantum ergodicity, quantum unique ergodicity, concentration of measure.

D. R. was partly supported by the grant "NOSEVOL" ANR-2011-BS01019 01.

L.T. was partly supported by the grant "HANDDY" ANR-10-JCJC 0109

and by the grant "ANAÉ" ANR-13-BS01-0010-03. 
Zelditch [22]) concerning the Laplace operator on compact Riemaniann manifolds. When the degree of $V$ tends to infinity, we formally recover the estimates of [2].

As a consequence of our probabilistic estimates, we obtain quantum ergodicity results for $\hat{H}$ under general probability laws satisfying the Gaussian concentration of measure property. These results extend previous ones [26, 2] obtained on compact manifolds for the Gaussian measure. Furthermore, our analysis allows to prove that almost all orthonormal bases of $L^{2}\left(\mathbb{R}^{d}\right)$ of Hermite functions are unique quantum ergodic (see Theorem 1.6 below).

Spectral theory context $:$ Let $d \geq 2$ and $k \geq 1$ an integer number and write $\langle x\rangle=\left(1+|x|^{2}\right)^{1 / 2}$. In the sequel, we will consider a potential $V$ satisfying

(A1) The potential $V$ is an elliptic polynomial in $\mathbb{R}^{d}$, which means that we have: $V=V_{0}+V_{1}$ where $V_{0}$ is an homogeneous polynomial of degree $2 k$ with $\left(V_{0}(x)>0\right.$ if $\left.x \neq 0\right)$ and $V_{1}(x)$ is a polynomial of degree $\leq 2 k-1$.

In particular for $|x|$ large we have $V(x) \approx\langle x\rangle^{2 k}$. Under these assumptions, $V$ is bounded from below; by adding a constant to $V$, we can assume that $V$ is nonnegative. Examples are $V(x)=|x|^{2 k}$ for $k \in \mathbb{N}^{*}$ or $V(x)=|x|^{4}+\alpha|x|^{2}, \alpha \in \mathbb{R}$.

Notice that we could have worked with more general smooth and confining potentials. We have chosen to restrict ourselves to assumption (A1) in order to lighten the proofs and to get explicit exponents.

Under these assumptions, $\hat{H}_{V}$ has a compact resolvent and its spectrum consists in a non decreasing sequence $\left\{\lambda_{j}\right\}_{j \geq 1}$ where each eigenvalue $\lambda_{j}$ is repeated according to its multiplicity and satisfies $\lim _{j \rightarrow+\infty} \lambda_{j}=+\infty$. Let $\left\{\varphi_{j}\right\}_{j \geq 1}$ be an orthonormal basis in $L^{2}\left(\mathbb{R}^{d}\right)$ of eigenfunctions of $\hat{H}_{V}$, $\hat{H}_{V} \varphi_{j}=\lambda_{j} \varphi_{j}$. Then one can show that $\varphi_{j}$ is in the Schwartz space $\mathcal{S}\left(\mathbb{R}^{d}\right)$ thanks to Agmon estimates.

For any interval $I$ of $\mathbb{R}$ we denote by $\Pi_{H}(I)$ the spectral projector of $\hat{H}$ on $I$. The range $\mathcal{E}_{H}(I)$ of $\Pi_{H}(I)$ is spanned by $\left\{\varphi_{j} ; \lambda_{j} \in I\right\}$ and $\Pi_{H}(I)$ has an integral kernel given by

$$
\pi_{H}(I ; x, y)=\sum_{\left[j: \lambda_{j} \in I\right]} \varphi_{j}(x) \overline{\varphi_{j}(y)} .
$$

If $\left.\left.I=I_{\lambda}:=\right] 0, \lambda\right]$, we denote by $\Pi(\lambda)=\Pi\left(I_{\lambda}\right), \mathcal{E}_{H}(\lambda)=\mathcal{E}_{H}\left(I_{\lambda}\right)$ and $\pi_{H}(\lambda ; x, y)=\pi_{H}(I ; x, y)$.

We have a "soft" Sobolev inequality (see e.g. [18, Lemma 3.1] for a proof):

$$
|u(x)| \leq\left(\pi_{H}(I ; x, x)\right)^{1 / 2}\|u\|_{L^{2}\left(\mathbb{R}^{d}\right)}, \quad \forall u \in \mathcal{E}_{H}(I) .
$$

In $\mathbb{R}^{d}$ the spectral function $\pi_{H_{V}}(\lambda ; x, x)$ is fast decreasing for $|x| \rightarrow+\infty$ so it is natural to replace the $L^{\infty}$ norm $\|u\|_{\infty}=\sup _{x \in \mathbb{R}^{d}}|u(x)|$ by the weighted norms $\|u\|_{\infty, s}=\sup _{x \in \mathbb{R}^{d}}\langle x\rangle^{s}|u(x)|$. 
The spectral theory for $\hat{H}$ has been studied in 4, 15, 10. In particular a Weyl asymptotics was proven:

$$
N_{H}(\lambda)=(2 \pi)^{-d} \int_{\left[|\xi|^{2}+V(x) \leq \lambda\right]} d \xi d x+\mathcal{O}\left(\lambda^{\frac{(d-1)(k+1)}{2 k}}\right), \lambda \rightarrow+\infty
$$

and using the assumptions $(\mathrm{A} 1)$ on $V$ we get $N_{H}(\lambda) \approx \lambda^{\frac{d(k+1)}{2 k}}$. We then consider the normalized Hamiltonian

$$
\hat{H}_{n o r}:=\hat{H}^{\frac{k+1}{2 k}} \text {, }
$$

so that we have

$$
N_{H_{\text {nor }}}(\lambda) \approx \lambda^{d} .
$$

The Sobolev spaces associated with $\hat{H}$ are here defined as follows. Let $s \geq 0, p \in[1,+\infty]$,

$$
\mathcal{W}_{k}^{s, p}:=\mathcal{W}_{k}^{s, p}\left(\mathbb{R}^{d}\right):=\left\{u \in L^{p}\left(\mathbb{R}^{d}\right), \hat{H}_{n o r}^{s} u \in L^{p}\left(\mathbb{R}^{d}\right)\right\},\|u\|_{s, p}=\left\|\hat{H}_{n o r}^{s} u\right\|_{L^{p}\left(\mathbb{R}^{d}\right)} .
$$

More explicitly, for $1<p<+\infty$ we can prove (see [24, Lemma 2.4])

$$
\mathcal{W}_{k}^{s, p}:=\mathcal{W}_{k}^{s, p}\left(\mathbb{R}^{d}\right):=\left\{u \in L^{p}\left(\mathbb{R}^{d}\right),(-\Delta)^{s \frac{k+1}{2 k}} u \in L^{p}\left(\mathbb{R}^{d}\right) \text { and }|x|^{s(k+1)} u \in L^{p}\left(\mathbb{R}^{d}\right)\right\} .
$$

The Hilbert Sobolev spaces are denoted by $\mathcal{H}_{k}^{s}=\mathcal{W}_{k}^{s, 2}$. The $\mathcal{H}_{k}^{s}$-norm is equivalent to the spectral norm

$$
\|u\|_{\mathcal{H}_{k}^{s}}=\left(\sum_{j \in \mathbb{N}} \lambda_{j}^{\frac{(k+1) s}{k}}\left|c_{j}\right|^{2}\right)^{1 / 2}, \text { when } \quad u(x)=\sum_{j \in \mathbb{N}} c_{j} \varphi_{j}(x) .
$$

Remark 1.1. - We stress that with the definition (1.5), $\hat{H}_{\text {nor }}$ is considered as an order 1 operator. This convention is different from the one used in [18, where the definitions of the Sobolev spaces were based on $\hat{H}$ instead of $\hat{H}_{\text {nor }}$.

Let $d \geq 2$. For $h>0$, we define the spectral interval $I_{h}=\left[\frac{a_{h}}{h}, \frac{b_{h}}{h}\left[\right.\right.$ and we assume that $a_{h}$ and $b_{h}$ satisfy, for some $a, b, D>0, \delta \in[0,1]$,

$$
\lim _{h \rightarrow 0} a_{h}=a, \quad \lim _{h \rightarrow 0} b_{h}=b, \quad 0<a \leq b \quad \text { and } \quad b_{h}-a_{h} \geq D h^{\delta} .
$$

with any $D>0$ if $\delta<1$ and $D$ some large constant if $\delta=1$. We denote by $N_{h}$ the number with multiplicities of eigenvalues of $\hat{H}_{n o r}$ in $I_{h}$. By (1.3) we have

$$
N_{h} \sim(2 \pi)^{-d} \int_{\frac{a_{h}}{h} \leq\left(|\xi|^{2}+V(x)\right)^{(k+1) /(2 k)}<\frac{b_{h}}{h}} d \xi d x .
$$

Then, under the previous assumptions, $\lim _{h \rightarrow 0} N_{h}=+\infty$.

In the sequel, we write $\Lambda_{h}=\left\{j \geq 1, \lambda_{j}^{\frac{k+1}{2 k}} \in I_{h}\right\}$ and $\mathcal{E}_{h}=\operatorname{span}\left\{\varphi_{j}, j \in \Lambda_{h}\right\}$, so that $N_{h}=\# \Lambda_{h}=$ $\operatorname{dim} \mathcal{E}_{h}$. We denote by $\mathbf{S}_{h}=\left\{u \in \mathcal{E}_{h}:\|u\|_{L^{2}\left(\mathbb{R}^{d}\right)}=1\right\}$ the unit sphere of $\mathcal{E}_{h}$. 
We will consider sequences of complex numbers $\gamma=\left(\gamma_{n}\right)_{n \in \mathbb{N}}$ so that there exists $K_{0}>0$

$$
\left.\left.\left|\gamma_{n}\right|^{2} \leq \frac{K_{0}}{N_{h}} \sum_{j \in \Lambda_{h}}\left|\gamma_{j}\right|^{2}, \quad \forall n \in \Lambda_{h}, \quad \forall h \in\right] 0,1\right] .
$$

This condition means that on each level of energy $\lambda_{n}, n \in \Lambda_{h}$, one coefficient $\left|\gamma_{k}\right|$ cannot be much larger than the others. Sometimes, in order to prove lower bound estimates, we will need the stronger condition $\left(K_{1}>0\right)$

$$
\left.\left.\frac{K_{1}}{N_{h}} \sum_{j \in \Lambda_{h}}\left|\gamma_{j}\right|^{2} \leq\left|\gamma_{n}\right|^{2} \leq \frac{K_{0}}{N_{h}} \sum_{j \in \Lambda_{h}}\left|\gamma_{j}\right|^{2}, \quad \forall n \in \Lambda_{h}, \quad \forall h \in\right] 0,1\right] .
$$

This condition which was suggested to us by Nicolas Burq, means that on each level of energy $\lambda_{n}, n \in \Lambda_{h}$, the coefficients $\left|\gamma_{k}\right|$ have almost the same size. For instance (1.9) holds if there exists $\left(d_{h}\right)_{h \in] 0,1]}$ so that $\gamma_{n}=d_{h}$ for all $n \in \Lambda_{h}$. These sequences will be used to built random wave packets $u_{\gamma}=\sum \gamma_{j} X_{j} \varphi_{j}$ for random variables $\left\{X_{j}\right\}_{j \geq 1}$.

Stochastic context : Consider a probability space $(\Omega, \mathcal{F}, \mathbb{P})$ and let $\left\{X_{n}, n \geq 1\right\}$ an i.i.d sequence of real or complex random variables with joint distribution $\nu$. In the sequel, we assume that the r.v. $X_{n}$ is centred $\left(\mathbb{E}\left(X_{n}\right)=0\right)$ and normalised $\left(\mathbb{E}\left(\left|X_{n}\right|^{2}\right)=1\right)$. For our results we will assume that $\nu$ satisfies a Gaussian concentration condition in the sense of the following definition:

Definition 1.2. - We say that a probability measure $\nu$ on $\mathbb{K}(\mathbb{K}=\mathbb{R}, \mathbb{C})$ satisfies the concentration of measure property if there exist constants $c, C>0$ independent of $N \in \mathbb{N}$ such that for all Lipschitz and convex function $F: \mathbb{K}^{N} \longrightarrow \mathbb{R}$

$$
\nu^{\otimes N}\left[X \in \mathbb{K}^{N}:|F(X)-\mathbb{E}(F(X))| \geq r\right] \leq c e^{-\frac{C r^{2}}{\|F\|_{L i p}^{2}}}, \quad \forall r>0,
$$

where $\|F\|_{\text {Lip }}$ is the best constant so that $|F(X)-F(Y)| \leq\|F\|_{\text {Lip }}\|X-Y\|_{\ell^{2}}$.

For instance, $\nu$ can be the standard normal law, a Bernoulli law or any law with bounded support (see [18, Section 2]). For details on this notion, see Ledoux [13].

Observe that if (1.10) is satisfied, that for $\varepsilon>0$ small enough, for $j \geq 1$ (see [18, (2.3)])

$$
\int_{\mathbb{K}} \mathrm{e}^{\varepsilon X_{j}^{2}} \mathrm{~d} \nu(x)<+\infty .
$$

If $\left(\gamma_{n}\right)_{n \in \mathbb{N}}$ satisfies (1.9) and $\nu$ satisfies (1.10), we define the random vector in $\mathcal{E}_{h}$

$$
v_{\gamma}(\omega):=v_{\gamma, h}(\omega)=\sum_{j \in \Lambda_{h}} \gamma_{j} X_{j}(\omega) \varphi_{j}
$$

and we define a probability $\mathbf{P}_{\gamma, h}$ on $\mathbf{S}_{h}$ by: for all measurable and bounded function $f: \mathbf{S}_{h} \longrightarrow \mathbb{R}$

$$
\int_{\mathbf{S}_{h}} f(u) \mathrm{d} \mathbf{P}_{\gamma, h}(u)=\int_{\Omega} f\left(\frac{v_{\gamma}(\omega)}{\left\|v_{\gamma}(\omega)\right\|_{L^{2}\left(\mathbb{R}^{d}\right)}}\right) \mathrm{d} \mathbb{P}(\omega) .
$$


In other words, $\mathbf{P}_{\gamma, h}$ is the push forward probability measure obtained by projection on the unit sphere of $\mathcal{E}_{h}$ of the law of the random vector $v_{\gamma}$. In the isotropic case $\left(\gamma_{j}=\frac{1}{\sqrt{N}}\right.$ for all $\left.j \in \Lambda_{h}\right)$, we denote this probability by $\mathbf{P}_{h}$. We can check (see e.g. [18, Appendix C]) that in the isotropic case and in the particular case where $\nu=\mathcal{N}_{\mathbb{C}}(0,1)$ or $\nu=\mathcal{N}_{\mathbb{R}}(0,1)$, then $\mathbf{P}_{h}$ (which we will denote by $\mathbf{P}_{h}^{(u)}$ ) is the uniform probability on $\mathbf{S}_{h}$.

Notice that if the probability law $\nu$ is rotation invariant on $\mathbb{C}$ then the probabilities $\mathbf{P}_{\gamma, h}$ are invariant by the Schrödinger linear flow $\mathrm{e}^{-i t h^{-1} \hat{H}}$.

1.2. Main results of the paper. - Let us state now the main results proved in this paper.

1.2.1. Estimates for frequency localised functions. - Our first result shows that for the elements in the support of $\mathbf{P}_{\gamma, h}$, the Sobolev estimates are better than usual. This phenomenon was studied in [2] on compact Riemannian manifolds and in [18] for the harmonic oscillator. Recall the definition (1.5) of the Sobolev spaces $\mathcal{W}_{k}^{s, p}\left(\mathbb{R}^{d}\right)$, then

Theorem 1.3. - Let $d \geq 2$ and assume that $0 \leq \delta<2 / 3$ in (1.6). Consider a potential $V$ which satisfies conditions (A1). Suppose that $\nu$ satisfies the concentration of measure property (1.10). Then there exist $0<C_{0}<C_{1}, c_{1}>0$ and $h_{0}>0$ such that for all $\left.\left.h \in\right] 0, h_{0}\right]$.

$$
\mathbf{P}_{\gamma, h}\left[u \in \mathbf{S}_{h}: C_{0}|\log h|^{1 / 2} \leq\|u\|_{\mathcal{W}_{k}^{\frac{d}{2(k+1)}, \infty}\left(\mathbb{R}^{d}\right)} \leq C_{1}|\log h|^{1 / 2}\right] \geq 1-h^{c_{1}} .
$$

Moreover the estimate from above holds true for any $0 \leq \delta \leq 1$ ( $D$ is large enough for $\delta=1$ ) and $\gamma$ satifying (1.8).

Recall that on the support of $\mathbf{P}_{\gamma, h}$ there exist $0<C_{2}<C_{3}$, so that for all $u \in \mathbf{S}_{h}$, and $s \geq 0$

$$
C_{2} h^{-s} \leq\|u\|_{\mathcal{H}_{k}^{s}\left(\mathbb{R}^{d}\right)} \leq C_{3} h^{-s},
$$

which shows that there is no random smoothing in the $L^{2}$ Sobolev scale spaces (see also Theorem 3.11). But, if $\hat{H}=-\Delta+V$ is considered as an operator of order 2, then using (1.4), Theorem 1.3 shows a gain of $d /(2 k)+d / 2$ derivatives in $L^{\infty}\left(\mathbb{R}^{d}\right)$ comparing with the deterministic Sobolev embedding. We shall see that this is really a stochastic smoothing property which has nothing to do with the usual Sobolev embedding.

For $k=1$ we recover [18, Theorem 1.1] in the case of the harmonic oscillator. In this particular case, we moreover construct Hilbertian bases of $L^{2}\left(\mathbb{R}^{d}\right)$ of eigenfunctions of $\hat{H}$ which enjoy good decay properties (see [18, Theorem 1.3]). For a general potential we can extend this result, with analogous bounds, but for quasimodes instead of exact eigenfunctions.

When $k \rightarrow+\infty$, the operator $\hat{H}$ seems to behave like the Laplacian on a compact manifold. In this case we get estimates close to the estimates of [2, Théorème 5].

The proof of Theorem 1.3 relies on a good understanding of the spectral function associated with $\hat{H}$, in particular on two weighted estimates in $L^{p}$ : The first is a semi-classical uniform upper bound obtained by Koch-Tataru-Zworski [12], while the second estimate is a lower bound which is proved in the Appendix. These results are combined with probabilistic techniques as in [2, 18]. 
1.2.2. Random quantum ergodicity. - Our second result concerns semi-classical measures associated with families of states $\left.\left.h \mapsto u_{h}, h \in\right] 0,1\right],\left\|u_{h}\right\|_{L^{2}\left(\mathbb{R}^{d}\right)}=1$. This development is inspired from [2], and can be interpreted as a kind of random-quantum-ergodicity (see [26]).

Assume that $\left.\left.J_{h}:=h I_{h}=\right] a_{h}, b_{h}\right]$ is such that

$$
\lim _{h \rightarrow 0} a_{h}=\lim _{h \rightarrow 0} b_{h}=\eta>0 \quad \text { and } \quad \lim _{h \rightarrow 0} \frac{b_{h}-a_{h}}{h}=+\infty .
$$

Denote by $L_{\eta}$ the Liouville measure associated with the classical Hamiltonian $H_{0}(x, \xi)=\frac{|\xi|^{2}}{2}+V_{0}(x)$ (recall that $V_{0}$ is defined in Assumption $(A 1)$ ). Then $L_{\eta}$ is given by

$$
L_{\eta}(A)=C_{\eta} \int_{\left[H_{0}(z)=\eta\right]} \frac{A(z)}{\left|\nabla H_{0}(z)\right|} d \Sigma_{\eta}(z)
$$

where $d \Sigma_{\eta}$ is the Euclidean measure on the hyper surface $\Sigma_{\eta}:=H_{0}^{-1}(\eta)$ and $C_{\eta}>0$ is a normalization constant such that $L_{\eta}$ is a probability measure on $\Sigma_{\eta}$.

We denote by $S(1, k)$ the class of symbols such that $A \in C^{\infty}\left(\mathbb{R}^{2 d}\right)$ and $A$ is quasi-homogeneous of degree 0 outside a small neighborhood of $(0,0)$ in $\mathbb{R}_{x}^{d} \times \mathbb{R}_{\xi}^{d}: A\left(\lambda x, \lambda^{k} \xi\right)=A(x, \xi)$ for every $\lambda \geq 1$ and $|(x, \xi)| \geq \varepsilon$.

For $A \in S(1, k)$ let us denote by $\hat{A}$ the Weyl quantization of $A$ (here $h=1$ ). Notice that if $\left\|u_{h}\right\|_{L^{2}\left(\mathbb{R}^{d}\right)}=1$ then $A \mapsto\left\langle u_{h}, \hat{A} u_{h}\right\rangle$ defines a semiclassical measure on $\Sigma_{\eta}$ (see e.g. [1]).

Then we have

Theorem 1.4. - Consider a potential $V$ which satisfies conditions (A1). Assume that we are in the isotropic case $\left(\gamma_{j}=\frac{1}{\sqrt{N_{h}}}\right.$ for all $\left.j \in \Lambda_{h}\right)$, and that $\nu$ satisfies the concentration of measure property (1.10). Then there exist $c, C>0$ so that for all $r \geq 1$ and $A \in S(1, k)$ with $\max _{(x, \xi) \in \Sigma_{1}}|A(x, \xi)| \leq 1$

$$
\mathbf{P}_{h}\left[u \in \mathbf{S}_{h}:\left|\langle u, \hat{A} u\rangle-L_{\eta}(A)\right|>r\right] \leq C e^{-c N_{h} r^{2}}
$$

By homogeneity we can also get an estimate for any $A \in S(1, k)$.

We are inspired from Burq-Lebeau [2, Théorème 3] who obtained a similar result for the Laplacian on a compact manifold. A modification of their proof allows to consider more general random variables satisfying the Gaussian concentration assumption instead of the uniform law.

The result of Theorem 1.4 can be related to quantum ergodicity (see [25, 3, 8]) which concerns the semi-classical behavior of $\left\langle\varphi_{j}, \hat{A} \varphi_{j}\right\rangle$ when the classical flow is ergodic on the energy hyper surface $\Sigma_{\eta}$. Then, for "almost all" eigenfunctions $\varphi_{j}$, we have $\left\langle\varphi_{j}, \hat{A} \varphi_{j}\right\rangle \stackrel{j \rightarrow+\infty}{\longrightarrow} L_{\eta}(A)$. The meaning of Theorem 1.4 is that we have $\langle u, \hat{A} u\rangle \stackrel{h \rightarrow 0}{\longrightarrow} L_{\eta}(A)$ for almost all $u \in \mathbf{S}_{h}$ such that all modes $\left(\varphi_{j}\right)_{j \in \Lambda_{h}}$ are "almostequi-present" in $u$. For related results see Zelditch [27.

As a consequence of Theorem 1.4 we easily get that almost all bases of Hermite functions are Quantum Uniquely Ergodic (see Theorem 1.6 for a precise statement). 
From (1.15) we directly deduce that there exists $C>0$ so that for all $p \geq 2$ and $h \in] 0,1]$,

$$
\left\|\langle u, \hat{A} u\rangle-L_{\eta}(A)\right\|_{L_{\mathbf{P}_{h}}^{p}} \leq C N_{h}^{-1 / 2} \sqrt{p}
$$

Therefore, if one denotes by

$$
u_{h}^{\omega}=\frac{1}{\sqrt{N_{h}}} \sum_{j \in \Lambda_{h}} X_{j}(\omega) \varphi_{j},
$$

then we have $\left\langle u_{h}^{\omega}, \hat{A} u_{h}^{\omega}\right\rangle \stackrel{h \rightarrow 0}{\longrightarrow} L_{\eta}(A)$ in $L^{p}(\Omega)$-norm with a remainder estimate. For example, we can apply this result to $u_{h}^{\omega}=\frac{1}{\sqrt{N_{h}}} \sum_{j \in \Lambda_{h}}(-1)^{\varepsilon_{j}(\omega)} \varphi_{j}$ where $\left\{\varepsilon_{j}\right\}_{j \geq 1}$ are i.i.d Bernoulli variables.

Remark 1.5. - It is likely that our main results can be extended in the case where $V$ is a smooth (instead of polynomial) confining potential, at the cost of some technicalities.

1.2.3. Quantum unique ergodicity of bases of Hermite functions. - As another application of the ergodicity results (see Proposition 4.1) we prove that random orthonormal basis of eigenfunctions of the Harmonic oscillator $\hat{H}=-\Delta+|x|^{2}$ is Quantum Uniquely Ergodic (QUE, according the terminology used in [27] and introduced in [23]).

Firstly, we assume that for all $j \in \Lambda_{h}, \gamma_{j}=N_{h}^{-1 / 2}$ and that $X_{j} \sim \mathcal{N}_{\mathbb{C}}(0,1)$, so that $\mathbf{P}_{h}:=\mathbf{P}_{\gamma, h}$ is the uniform probability on $\mathbf{S}_{h}$. Set $\eta=2$, then the Liouville measure $L_{2}$ is the uniform measure on $\left\{(x, \xi) \in \mathbb{R}^{2 d}:|x|^{2}+|\xi|^{2}=2\right\}=\sqrt{2} \mathbb{S}^{2 d-1}$. We set $h_{j}=1 / j$ with $j \in \mathbb{N}^{*}$, and

$$
a_{h_{j}}=2+d h_{j}, \quad b_{h_{j}}=2+(2+d) h_{j} .
$$

Then (1.6) is satisfied with $\delta=1$ and $D=2$. In particular, each interval

$$
I_{h_{j}}=\left[\frac{a_{h_{j}}}{h_{j}}, \frac{b_{h_{j}}}{h_{j}}[=[2 j+d, 2 j+d+2[\right.
$$

only contains the eigenvalue $2 j+d$ with multiplicity $N_{h_{j}} \sim c j^{d-1}$, and $\mathcal{E}_{h_{j}}$ is the corresponding eigenspace of the harmonic oscillator $H$. We can identify the space of the orthonormal basis of $\mathcal{E}_{h_{j}}$ with the unitary group $U\left(N_{h_{j}}\right)$ and we endow $U\left(N_{h_{j}}\right)$ with its Haar probability measure $\rho_{j}$. Then the space $\mathcal{B}$ of the Hilbertian bases of eigenfunctions of $H$ in $L^{2}\left(\mathbb{R}^{d}\right)$ can be identified with

$$
\mathcal{B}=\times_{j \in \mathbb{N}} U\left(N_{h_{j}}\right),
$$

which can be endowed with the measure

$$
\mathrm{d} \rho=\otimes_{j \in \mathbb{N}} \mathrm{d} \rho_{j} .
$$

Denote by $B=\left(\varphi_{j, \ell}\right)_{j \in \mathbb{N}, \ell \in \llbracket 1, N_{h_{j}} \rrbracket} \in \mathcal{B}$ a typical orthonormal basis of $L^{2}\left(\mathbb{R}^{d}\right)$ so that for all $j \in \mathbb{N}$, $\left(\varphi_{j, \ell}\right)_{\ell \in \llbracket 1, N_{h_{j}} \rrbracket} \in U\left(N_{h_{j}}\right)$ is an orthonormal basis of $\mathcal{E}_{h_{j}}$.

Theorem 1.6. - Let $\hat{H}$ be the harmonic oscillator. For $B \in \mathcal{B}$ and $A \in S(1,1)$ denote by

$$
D_{j}(B)=\max _{1 \leq \ell \leq N_{h_{j}}}\left|\left\langle\varphi_{j, \ell}, \hat{A} \varphi_{j, \ell}\right\rangle-L_{2}(A)\right| .
$$


Then we have

$$
\lim _{j \rightarrow+\infty} D_{j}(B)=0, \quad \rho-\text { a.s on } \mathcal{B} .
$$

In other words, a random orthonormal basis of Hermite functions is QUE.

In [26] the author proved that on the standard sphere a random orthonormal basis of eigenfunctions of the Laplace operator is ergodic. In [14 the author get a unique quantum ergodicity result for compact Riemannian manifolds.

Using estimates proved in [2], an analogous result to Theorem 1.6 can be obtained for the Laplace operator on Riemannian compact manifolds with the same method. This holds true in particular for the sphere in any dimension $d \geq 2$ and more generally for Zoll manifolds (in this last setting a random orthonormal basis of quasi-modes is obtained).

1.3. Plan of the paper. - The rest of the paper is organised as follows. In Section 2 we state crucial estimates on the spectral function which are used in Section 3 for the proof of Theorem 1.3. Section 4 is devoted to the proof of Theorem 1.4. Finally, in the appendix, we prove some point-wise estimates for the spectral function.

Acknowledgements. - The authors benefited from discussions with Nicolas Burq and Aurélien Poiret.

\section{Estimates for the spectral function}

Our goal here is to obtain for the spectral function of operators $\hat{H}$ satisfying (A1), analogous estimates as those proved in [11] and in [12 for the harmonic oscillator. These estimates are more or less known, in particular some are contained in [11, but they are crucial for proving our first main result, so we state them in a form suitable for us. The difficulties to prove them increase with the parameter $\delta \in[0,1]$ measuring the width of the spectral window $I_{h}$. Concerning upper bounds, the case $\delta=0$ is easy; $\delta<2 / 3$ can be reached using accurate properties of the semi-classical Weyl calculus; the case $\delta=1$ was solved in [12] using a semi-classical Strichartz estimate. However, for the lower bounds, we need to assume $\delta<2 / 3$.

2.1. An upper bound of the spectral function. - Here we cannot use explicit formulas like the Mehler formula for the harmonic oscillator. But it is possible to use a global pseudo-differential calculus to construct a parametrix for $(\hat{H}-\lambda)^{-1}$ where $\lambda \in \mathbb{C}, \lambda \notin[0, \infty[$ and to use some estimates proved in [19. We give in Appendix A.2 the main steps to prove the following result.

Proposition 2.1. - Let us denote by $K_{H}(t ; x, y)$ the heat kernel of $\hat{H}=-\Delta+V$. Then for every $N \geq 1$ there exists $C_{N}>0$ such that

$$
K_{H}(t ; x, x) \leq C_{N}\left(t^{-d / 2} \exp (-t V(x))+\left(1+|x|^{2 k}\right)^{-N}\right)
$$

for every $t \in] 0,1]$ and $x \in \mathbb{R}^{d}$. 
The bound stated in Proposition 2.1 is relevant for $t \rightarrow 0$. For better estimates in the regime $t>0$ we refer e.g. to [21, Proposition 1] and references therein.

We get easily the following estimate for the spectral function of $\hat{H}$ defined in (1.1).

Corollary 2.2. - With the notations of the previous Proposition we have

$$
\pi_{H}(\lambda ; x, x) \leq C_{N}\left(\lambda^{d / 2} \exp (-V(x) / \lambda)+\left(1+|x|^{2 k}\right)^{-N}\right), \quad \forall \lambda \geq 1 .
$$

In particular for every $\theta \geq 0$ we have

$$
\pi_{H}(\lambda ; x, x) \leq C \lambda^{(d+\theta) / 2}\langle x\rangle^{-k \theta}
$$

and for every $u \in \mathcal{E}_{H}(\lambda)$, thanks to (1.2) we have

$$
\langle x\rangle^{k \theta / 2}|u(x)| \leq C_{\theta} \lambda^{\frac{d+\theta}{4}}\|u\|_{L^{2}\left(\mathbb{R}^{d}\right)} .
$$

Remark 2.3. - Recall that $N_{H}(\lambda)$ (number of eigenvalues $\leq \lambda$ ) is of order $\lambda^{d_{k}}$ with $d_{k}=\frac{d(k+1)}{2 k}$. We shall see that $\theta=\frac{d}{k}$ is specific because we get from (2.1)

$$
\langle x\rangle^{d / 2}|u(x)| \leq C \lambda^{\frac{d_{k}}{2}}\|u\|_{L^{2}\left(\mathbb{R}^{d}\right)} .
$$

Notice that we also have

$$
\pi_{H}(\lambda ; x, x) \leq C \lambda^{d / 2}
$$

Now using the definition of $h=\lambda^{-\frac{k+1}{2 k}}$ we get that

$$
\langle x\rangle^{k \theta / 2} h^{\frac{k(d+\theta)}{2(k+1)}}|u(x)| \leq C\|u\|_{L^{2}\left(\mathbb{R}^{d}\right)}, \quad \forall u \in \mathcal{E}_{H_{\text {nor }}}\left(h^{-1}\right) .
$$

2.2. A uniform estimate for the spectral function. - Now we shall state more refined estimates. Let $V$ be a potential satisfying (A1), then we have

Proposition 2.4. - For every $\delta \in[0,1]$ and $C_{0}>0$, there exists $C>0$ such that for every $\theta \in\left[0, \frac{d}{k}\right]$ and $1 \leq r \leq \infty$

$$
\left\|\pi_{H_{n o r}}(\lambda+\mu ; x, x)-\pi_{H_{n o r}}(\lambda ; x, x)\right\|_{L^{r, k(r-1) \theta}\left(\mathbb{R}^{d}\right)} \leq C \lambda^{\alpha}
$$

for $|\mu| \leq C_{0} \lambda^{1-\delta}, \lambda \geq 1$ and with $\alpha=\frac{d}{k+1}\left(k+\frac{1}{r}\right)-\delta+\frac{k \theta}{k+1}\left(1-\frac{1}{r}\right)$, where $\|u\|_{L^{r, s}\left(\mathbb{R}^{d}\right)}^{r}=\int_{\mathbb{R}^{d}}\langle x\rangle^{s}|u(x)|^{r} d x$.

The proof Proposition 2.4 is a consequence of the following more general semiclassical result, which is proved in Appendix A.3, See also Ivrii [10] for related work and [18, Lemma 3.5] for more details. For $0<\varepsilon<1$, denote by $V_{\varepsilon}(x)=\varepsilon^{2 k} V(x / \varepsilon)$, where $V$ satisfies (A1) and consider the operator $\hat{H}_{\varepsilon}(h)=-h^{2} \Delta+V_{\varepsilon}$. Then

Proposition 2.5. - For every $\delta \in[0,1]$, every $C_{0}>0$ there exist $\varepsilon_{0}>0$ and $C_{1}>0$ such that for every $\left.\nu \in] \nu_{0}-\varepsilon_{0}, \nu_{0}+\varepsilon_{0}\left[,|\mu| \leq C_{0} h^{\delta-1}, x \in \mathbb{R}^{d}, h \in\right] 0,1\right]$ and $\left.\left.\varepsilon \in\right] 0,1\right]$ we have

$$
\left|\pi_{H_{\varepsilon}(h)}(\nu+\mu h ; x, x)-\pi_{H_{\varepsilon}(h)}(\nu ; x, x)\right| \leq C_{1} h^{\delta-d} .
$$

Proof. — See Appendix A.3. 
Remark 2.6. - Actually, this result holds true for a larger class of potentials $\left(V_{\varepsilon}\right)_{\varepsilon \in(0,1]} \subset \mathcal{C}^{\infty}\left(\mathbb{R}^{d}\right)$, which satisfy the following conditions: Let $\nu_{0} \in \mathbb{R}, \varepsilon_{0}>0$ and denote by $I_{0}=\left[\nu_{0}-\varepsilon_{0}, \nu_{0}+\varepsilon_{0}\right]$. We assume that there exists a compact $K \subset \mathbb{R}^{d}$ so that, uniformly in $\varepsilon \in(0,1]$

$$
\begin{cases}\text { - } & V_{\varepsilon}^{-1}\left(I_{0}\right) \subset K \\ \text { - }\left|\partial_{x}^{\alpha} V_{\varepsilon}(x)\right| \leq C_{\alpha} \text { for all } x \in K \text { and } \alpha \in \mathbb{N}^{d} \\ \text { - There exists } \delta_{0}>0 \text { so that }\left|\nabla V_{\varepsilon}(x)\right| \geq \delta_{0} \text { for all } x \in K\end{cases}
$$

Remark 2.7. - As we can see in Appendix A.3, the proof uses standard semiclassical arguments, with the semiclassical parameter $h \approx \lambda^{-\frac{k+1}{2 k}}$, where $\lambda$ is the natural energy parameter of our initial Hamiltonian $H_{V}$ (here $2 k$ is the degree of homogeneity of $V$ ).

As already said, for $0 \leq \delta<2 / 3$ the Proposition can be proved using semiclassical pseudo-differential Weyl calculus. The general case $0 \leq \delta \leq 1$ is more difficult(1). We shall see in Appendix that it is a consequence of results proved in [12] extended to Hamiltonians depending smoothly on a parameter $\varepsilon$.

Proof of Proposition 2.4. - We reduce the problem to a semi-classical estimate.

Let us consider the eigenvalue problem

$$
\left(-\Delta_{x}+V(x)\right) \varphi_{j}(x)=\lambda_{j} \varphi_{j}(x) .
$$

Consider the $\left(\psi_{j}^{h}\right)$ defined by

$$
\psi_{j}^{h}(y)=h^{-\frac{d}{2(k+1)}} \varphi_{j}\left(h^{-\frac{1}{k+1}} y\right) .
$$

Performing the change of variable $x=y h^{-\frac{1}{k+1}}$, equation (2.3) is transformed into

$$
\left(-h^{2} \Delta_{y}+V_{\varepsilon}(y)\right) \psi_{j}^{h}(y)=\lambda_{j}(h) \psi_{j}^{h}(y),
$$

$\lambda_{j}(h)=\lambda_{j} h^{\frac{2 k}{k+1}}$, and where $V_{\varepsilon}$ is the potential

$$
V_{\varepsilon}(y)=\varepsilon^{2 k} V\left(\frac{y}{\varepsilon}\right),
$$

which is smooth in the parameter $\varepsilon \in[0,1]$ and is computed for $\varepsilon=h^{\frac{1}{k+1}}$.

So $\lambda \leq \lambda_{j}^{\frac{k+1}{2 k}} \leq \lambda+\mu$ if and only if $\lambda h \leq\left(\lambda_{j}(h)\right)^{\frac{k+1}{2 k}} \leq \lambda h+\mu h$, therefore we can fix a large real parameter $\tau$ and work with the semiclassical parameter $h=\frac{\tau}{\lambda}$ which is small for $\lambda$ large. Denote by $\hat{H}_{\varepsilon}(h)=-h^{2} \Delta+V_{\varepsilon}(x)$. Then the potential $V_{\varepsilon}$ satisfies the condition (A1) and we can apply Proposition 2.5. We deduce that for every $c>0$ and for every $\delta \leq 1$ there exists $C>0$ such that

$$
\left|\pi_{H_{\text {nor }}}(\lambda+\mu ; x, x)-\pi_{H_{\text {nor }}}(\lambda ; x, x)\right| \leq C \lambda^{d \frac{k}{k+1}-\delta}
$$

for every $\lambda>0,0 \leq \mu \leq c \lambda^{1-\delta}$.

Moreover we get that for every $\delta \in[0,1]$ and $C_{0}>0$, there exists $C>0$ such that for every $\theta \in\left[0, \frac{d}{k}\right]$

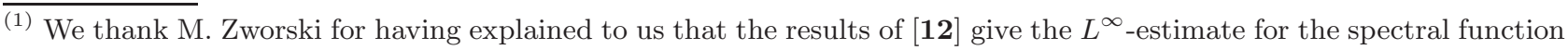
with $\delta=1$, in particular at the turning points.
} 
there exists $C>0$ such that

$$
\left|\pi_{H_{n o r}}(\lambda+\mu ; x, x)-\pi_{H_{n o r}}(\lambda ; x, x)\right| \leq C \lambda^{\frac{k(d+\theta)}{k+1}-\delta}\langle x\rangle^{-k \theta}
$$

for $|\mu| \leq C_{0} \lambda^{1-\delta}, \lambda \geq 1$.

It is enough to prove (2.5) for $|x| \leq C \lambda^{\frac{1}{k+1}}$ with $C>0$ large enough, using that $\pi_{H}(\lambda ; x, x)=$ $\pi_{H_{n o r}}\left(\lambda^{\frac{2 k}{k+1}} ; x, x\right)$ and that for $\langle x\rangle^{2 k} \gtrsim \lambda, \pi_{H}(\lambda ; x, x)$ is fast decreasing in $x$ and $\lambda$. For $|x| \leq C \lambda^{\frac{1}{k+1}}$, the bound (2.5) is clear.

Finally, the inequality (2.2) is a consequence of an interpolation inequality and (2.5).

\section{Probabilistic weighted Sobolev estimates}

Our main goal here is to prove Theorem 1.3. We follow the strategy initiated in 2 and worked out in 18 for the harmonic weighted spaces. A key step for the extension to potentials satisfying (A1), is to obtain suitable spectral estimates. In particular the probabilistic tools (concentration of measures) can be applied in a general setting as we have explained in [18] and will be used freely here, so we shall not give all the details in the proofs.

Here we shall denote by $h=\lambda^{-1}$ ( $\lambda$ is a typical energy of the rescaled Hamiltonian $\hat{H}_{\text {nor }}$ ). Using the Weyl asymptotic formula (1.3) we can infer that there exist $h_{0}>0, c>0, C>0$ such that

$$
\left.\left.c h^{-d}\left(b_{h}-a_{h}\right) \leq N_{h} \leq C h^{-d}\left(b_{h}-a_{h}\right), \quad \forall h \in\right] 0, h_{0}\right] .
$$

We are using the same notations as in the introduction for the Hamiltonian $\hat{H}_{n o r}$. In particular $N_{h}$ is the number of eigenvalues of $\hat{H}_{\text {nor }}$ in $\left.I_{h}=\right] \frac{a_{h}}{h}, \frac{b_{h}}{h}[$.

We are interested in Sobolev type estimates for $u$ in the spectral subspace $\mathcal{E}_{h}:=\Pi_{H_{\text {nor }}}\left(I_{h}\right)\left(L^{2}\left(\mathbb{R}^{d}\right)\right)$.

Following the strategy of [2], repeated in [18, we need to estimate $\frac{e_{x, h}}{N_{h}}$ where

$$
e_{x, h}=\pi_{H_{\text {nor }}}\left(\frac{b_{h}}{h} ; x, x\right)-\pi_{H_{\text {nor }}}\left(\frac{a_{h}}{h} ; x, x\right) .
$$

Lemma 3.1. - For every $\delta \in[0,1]$ there exist $C>0$ and $h_{0}>0$ such that we have

$$
\left.\left.e_{x, h} \leq C N_{h} h^{\frac{d-k \theta}{k+1}}\langle x\rangle^{-k \theta}, \quad \forall h \in\right] 0, h_{0}\right], \quad \forall \theta \in\left[0, \frac{d}{k}\right] .
$$

Proof. - To avoid some misunderstanding recall here the meaning of the parameters $\lambda$ and $h$. If for $H$ the typical energy is $\lambda$ then for $H_{n o r}$ the typical energy is $\lambda_{\text {nor }}$ where we have $\lambda_{\text {nor }}=\lambda^{\frac{k+1}{2 k}}$. Finally we have $h=\lambda_{\text {nor }}^{-1}$. So, we have

$$
e_{x, h}=\pi_{H}\left(\left(\frac{b_{h}}{h}\right)^{2 k /(k+1)} ; x, x\right)-\pi_{H}\left(\left(\frac{a_{h}}{h}\right)^{2 k /(k+1)} ; x, x\right),
$$

and (3.2) is a consequence of (3.1) and (2.5) . 
As for the harmonic oscillator we get, using (1.2) and interpolation, for every $u \in \mathcal{E}_{h}, \theta \geq 0, p \geq 2$

$$
\begin{array}{r}
\|u\|_{L^{\infty, k \theta / 2}\left(\mathbb{R}^{d}\right)} \leq C\left(N_{h} h^{\frac{d-k \theta}{k+1}}\right)^{1 / 2}\|u\|_{L^{2}\left(\mathbb{R}^{d}\right)} \\
\|u\|_{L^{p, k \theta(p / 2-1)\left(\mathbb{R}^{d}\right)}} \leq C\left(N_{h} h^{\frac{d-k \theta}{k+1}}\right)^{\frac{1}{2}-\frac{1}{p}}\|u\|_{L^{2}\left(\mathbb{R}^{d}\right)},
\end{array}
$$

where $L^{r, s}\left(\mathbb{R}^{d}\right):=L^{r}\left(\mathbb{R}^{d},\langle x\rangle^{s} d x\right)$ for $s \in \mathbb{R}, r \geq 1$. Notice that $N_{h}$ is of order $\left(b_{h}-a_{h}\right) h^{-d}$.

3.1. Upper bounds. — We can get now the following result.

Theorem 3.2. - There exist $\left.\left.h_{0} \in\right] 0,1\right], c_{2}>0$ and $C>0$ such that if $c_{1}=d(1+d / 2)$ we have

$$
\left.\left.\mathbf{P}_{\gamma, h}\left[u \in \mathbf{S}_{h}, h^{-\frac{d-k \theta}{2(k+1)}}\left\|\langle x\rangle^{k \theta / 2} u\right\|_{\infty}>\Lambda\right] \leq C h^{-c_{1}} \mathrm{e}^{-c_{2} \Lambda^{2}}, \forall \Lambda>0, \forall h \in\right] 0, h_{0}\right], \forall \theta \in\left[0, \frac{d}{k}\right] .
$$

Moreover we can choose every $c_{2}<\ell_{\theta}$ and $h_{0}$ small enough where

$$
\ell_{\theta}=\liminf _{h \searrow 0} \inf _{x \in \mathbb{R}^{d}}\left(\frac{N_{h}-1}{e_{x, h}} h^{\frac{d-k \theta}{k+1}}\langle x\rangle^{-k \theta}\right),
$$

and $\inf _{\theta \in[0, d / k]} \ell_{\theta}>0$.

Proof. - Similarly to [18, Theorem 4.1], we first show that there exists $c_{2}>0$ such that for every $\theta \in\left[0, \frac{d}{k}\right]$ every $x \in \mathbb{R}^{d}$, and every $\Lambda>0$ we have

$$
\mathbf{P}_{\gamma, h}\left[u \in \mathbf{S}_{h},\langle x\rangle^{\frac{k \theta}{2}} h^{-\frac{d-k \theta}{2(k+1)}}|u(x)|>\Lambda\right] \leq \mathrm{e}^{-c_{2} \Lambda^{2}},
$$

then a partition of unity argument yields the result. The fact that $\ell_{\theta}>0$ follows from (3.2).

So we get probabilistic Sobolev estimates improving the deterministic one (3.3) with probability close to one as $h \rightarrow 0$. The improvement is "almost" of order $N_{h}^{1 / 2} \approx\left(\left(b_{h}-a_{h}\right) h^{-d}\right)^{1 / 2}$. Choosing $\Lambda=\sqrt{-K \log h}$ we get the next result

Corollary 3.3. - For every $K>c_{1} / c_{2}$ we have

$$
\left.\left.\mathbf{P}_{\gamma, h}\left[u \in \mathbf{S}_{h},\|u\|_{L^{\infty, k \theta / 2}\left(\mathbb{R}^{d}\right)}>|K \log h|^{1 / 2} h^{\frac{d-k \theta}{2(k+1)}}\right] \leq h^{K c_{2}-c_{1}}, \quad \forall h \in\right] 0, h_{0}\right], \quad \forall \theta \in\left[0, \frac{d}{k}\right] .
$$

and

$$
\left.\left.\mathbf{P}_{\gamma, h}\left[u \in \mathbf{S}_{h}:\|u\|_{\mathcal{W}_{k}^{s, \infty}\left(\mathbb{R}^{d}\right)}>K h^{\frac{d}{2(k+1)}-s}|\log h|^{1 / 2}\right] \leq h^{K c_{2}-c_{1}}, \quad \forall h \in\right] 0, h_{0}\right], \forall s \geq 0 .
$$

Using the same argument that in [18, Section 3.1] we can deduce from the last corollary a global probabilistic Sobolev estimate. Let $\theta$ a $C^{\infty}$ real function on $\mathbb{R}$ such that $\theta(t)=0$ for $t \leq a, \theta(t)=1$ for $t \leq b / 2$ with $0<a<b / 2$. Define $\psi_{-1}(t)=1-\theta(t), \psi_{j}(t)=\theta\left(h_{j} t\right)-\theta\left(h_{j+1} t\right)$ for $j \in \mathbb{N}$. For every distribution $u \in \mathcal{S}^{\prime}\left(\mathbb{R}^{d}\right)$ we introduce the following dyadic Littlewood-Paley decomposition $\left(h_{j}=2^{-j}\right)$,

$$
u=\sum_{j \geq-1} u_{j}, \quad \text { with } \quad u_{j}=\sum_{\ell \in \mathbb{N}} \psi_{j}\left(\lambda_{\ell}\right)\left\langle u, \varphi_{\ell}\right\rangle \varphi_{\ell}
$$


and we have $u_{j} \in \mathcal{E}_{h_{j}}$.

The following scale of spaces contains the Besov spaces $B_{2, \infty}^{s}$ for every $s>0$ and $m \geq 0$.

$$
\mathcal{G}^{m}=\left\{u \in \mathcal{S}^{\prime}\left(\mathbb{R}^{d}\right): \sum_{j \geq 1} j^{m}\left\|u_{j}\right\|_{L^{2}\left(\mathbb{R}^{d}\right)}<+\infty\right\}, \quad m \geq 0 .
$$

We introduce probabilities $\mu_{\gamma}^{m}$ on $\mathcal{G}^{m}$ (see [18] for details) as the law of $v_{\gamma}(\omega)=\sum_{j \geq 0} \gamma_{j} X_{j}(\omega) \varphi_{j}$. In particular we can manage such that $\mu_{\gamma}^{1 / 2}\left(\mathcal{G}^{1 / 2}\right)=1$ and $\mu_{\gamma}^{1 / 2}\left(\mathcal{G}^{m}\right)=0$ for $m>1 / 2$.

Corollary 3.4. - There exists $c>0$ such that for every $\kappa>0$ large enough, there exists a Borel subset $B^{\kappa}$ of $\mathcal{G}^{1 / 2}$ with $\mu_{\gamma}^{1 / 2}\left(B^{\kappa}\right) \geq 1-\mathrm{e}^{-c \kappa^{2}}$, such that for every $u \in B^{\kappa}$ we have

$$
\|u\|_{\mathcal{W}_{k}^{\frac{d}{2(k+1)}, \infty}{ }_{\left(\mathbb{R}^{d}\right)}} \leq \kappa\|u\|_{\mathcal{G}^{1 / 2}} .
$$

Here, when $k \rightarrow \infty$, we formally recover the estimates proved by Burq-Lebeau [2, Corollaire 1.1] in case of a compact manifold.

3.2. Lower bounds. - Here we suppose that the random variables follow the Gaussian law $\mathcal{N}_{\mathbb{C}}(0,1)$ and that $\delta<2 / 3$.

Now we are interested to get a lower bound for $\|u\|_{L^{\infty, k \theta / 2}\left(\mathbb{R}^{d}\right)}$ and $\|u\|_{\mathcal{W}_{k}^{s, \infty}}$. As we have seen in [18, a first step is to get two sides weighted $L^{r}$ estimates for large $r$.

Our goal now is to prove the following probabilistic improvement of (3.3) when $\theta=d / k$

Theorem 3.5. - There exist $0<K_{0}<K_{1}, K>0, c_{1}>0, h_{0}>0$ such that

$$
\mathbf{P}_{\gamma, h}\left[u \in \mathbf{S}_{h}:\left|\|u\|_{L^{r, \frac{d}{h}\left(\frac{r}{2}-1\right)}}-\mathcal{M}_{r}\right|>\Lambda\right] \leq 2 \exp \left(-c_{2} N_{h}^{2 / r} \Lambda^{2}\right),
$$

for all $r \in[2, K|\log h|]$ and $\left.h \in] 0, h_{0}\right]$.

Therefore for every $\kappa \in] 0,1\left[, K>0\right.$, there exist $0<C_{0}<C_{1}, c_{1}>0, h_{0}>0$ such that for all $\left.\left.r \in\left[2, K|\log h|^{\kappa}\right], h \in\right] 0, h_{0}\right]$ and $\Lambda>0$ we have

$$
\mathbf{P}_{\gamma, h}\left[u \in \mathbf{S}_{h}: C_{0} \sqrt{r} \leq\|u\|_{L^{r, \frac{d}{k}\left(\frac{r}{2}-1\right)}} \leq C_{1} \sqrt{r}\right] \geq 1-\mathrm{e}^{-c_{1}|\log h|^{1-\kappa}} .
$$

Thus for $\theta=d / k$ we get a two sides weighted $L^{\infty}$ estimate showing that Theorem 3.2 and its corollary are sharp.

Corollary 3.6. - After a slight modification of the constants in Theorem 3.5, if necessary, we get

$$
\mathbf{P}_{\gamma, h}\left[u \in \mathbf{S}_{h}: K_{0}|\log h|^{1 / 2} \leq\|u\|_{L^{\infty, d / 2}} \leq K_{1}|\log h|^{1 / 2}\right] \geq 1-h^{c_{1}}
$$

for all $\left.h \in] 0, h_{0}\right]$.

The key ingredient of the proof is a two side estimate of a weighted norm of the spectral function. More precisely, we have the following result, which will be proven in Appendix A.4. 
Lemma 3.7. - There exist $0<C_{0}<C_{1}$ and $h_{0}>0$ such that

$$
C_{0} N_{h} h^{\beta_{2 p, \theta}} \leq\left(\int_{\mathbb{R}^{d}}\langle x\rangle^{k \theta(p-1)} \mathrm{e}_{x, h}^{p} d x\right)^{1 / p} \leq C_{1} N_{h} h^{\beta_{2 p, \theta}},
$$

for every $p \in\left[1, \infty[\right.$ and $\left.h \in] 0, h_{0}\right]$ where $\beta_{r, \theta}=\frac{d-k \theta}{k+1}\left(1-\frac{2}{r}\right)$.

Set $F_{r}(u)=\|u\|_{L^{r, \theta(r / 2-1)}}$ and denote by $\mathcal{M}_{r}$ its median, and by $\mathcal{A}_{r}^{r}=\mathbf{E}_{h}\left(F_{r}^{r}\right)$ the moment of order $r$. From Lemma 3.7 we get the estimates (see the proof of [18, Theorem 4.7])

$$
\left.\left.C_{0} \sqrt{r} h^{\beta_{r, \theta}} \leq \mathcal{M}_{r}, \mathcal{A}_{r} \leq C_{1} \sqrt{r} h^{\beta_{r, \theta}}, \quad \forall h \in\right] 0, h_{0}\right], \quad r \in\left[2, K \log N_{h}\right] .
$$

In particular, for $\theta=\frac{d}{k}$ we get

$$
\left.\left.C_{0} \sqrt{r} \leq \mathcal{M}_{r}, \mathcal{A}_{r} \leq C_{1} \sqrt{r}, \quad \forall h \in\right] 0, h_{0}\right], \quad r \in\left[2, K \log N_{h}\right],
$$

so Theorem 3.5 and its corollary follow from concentration of measures properties.

Remark 3.8. - For the median $\mathcal{M}_{\infty}$ and the mean $\mathcal{A}_{\infty}$ of $F_{\infty}(u):=\|u\|_{L^{\infty, d / 2}}$ we get the estimates

$$
\left.\left.K_{0}|\log h|^{1 / 2} \leq \mathcal{M}_{\infty}, \mathcal{A}_{\infty} \leq K_{1}|\log h|^{1 / 2}, \quad \forall h \in\right] 0, h_{0}\right] .
$$

We now state analogous results for the norm in the Sobolev scale $\mathcal{W}_{k}^{s, r}$ for which Theorem 1.3 is a particular case. Denote by $\mathcal{M}_{r, s}$ a median of $\|u\|_{\mathcal{W}_{k}^{s, r}\left(\mathbb{R}^{d}\right)}$ and set $\beta_{s}=\frac{d}{2(k+1)}\left(1-\frac{2}{r}\right)-s$. Then

Theorem 3.9. - Let $s \geq 0$. There exist $0<C_{0}<C_{1}, K>0, c_{1}>0, h_{0}>0$ such that for all $r \in[2, K|\log h|]$ and $\left.h \in] 0, h_{0}\right]$

$$
\mathbf{P}_{\gamma, h}\left[u \in \mathbf{S}_{h}:\left|\|u\|_{\mathcal{W}_{k}^{s, r}\left(\mathbb{R}^{d}\right)}-\mathcal{M}_{r, s}\right|>\Lambda\right] \leq 2 \exp \left(-c_{2} N_{h}^{2 / r} h^{-2 \beta_{s}} \Lambda^{2}\right)
$$

and

$$
C_{0} \sqrt{r} h^{\beta_{s}} \leq \mathcal{M}_{r, s} \leq C_{1} \sqrt{r} h^{\beta_{s}}, \quad \forall r \in[2, K \log N] .
$$

In particular, for every $\kappa \in] 0,1\left[, K>0\right.$, there exist $C_{0}>0, C_{1}>0, c_{1}>0$ such that for every $r \in\left[2, K|\log h|^{\kappa}\right]$ we have

$$
\mathbf{P}_{\gamma, h}\left[u \in \mathbf{S}_{h}: C_{0} \sqrt{r} h^{\frac{d}{2(k+1)}\left(1-\frac{2}{r}\right)} h^{-s} \leq\|u\|_{\mathcal{W}_{k}^{s, r}\left(\mathbb{R}^{d}\right)} \leq C_{1} \sqrt{r} h^{\frac{d}{2(k+1)}\left(1-\frac{2}{r}\right)} h^{-s}\right] \geq 1-\mathrm{e}^{-c_{1}|\log h|^{1-\kappa}},
$$

and for $r=+\infty$ we have for all $\left.h \in] 0, h_{0}\right]$

$$
\mathbf{P}_{\gamma, h}\left[u \in \mathbf{S}_{h}: C_{0}|\log h|^{1 / 2} h^{\frac{d}{2(k+1)}}-s=\|u\|_{\mathcal{W}_{k}^{s, \infty}\left(\mathbb{R}^{d}\right)} \leq C_{1}|\log h|^{1 / 2} h^{\frac{d}{2(k+1)}}-s\right] \geq 1-h^{c_{1}} .
$$

Remark 3.10. - For more general probability laws satisfying the Gaussian concentration estimate we can get a weaker lower bound as in [18, Theorem 4.13]. 
3.3. No random smoothing in $L^{2}$-scale spaces. - This subsection is a generalization to non harmonic potentials of results proved by Poiret [17, Section 4], and we refer to this paper for more details.

Let $v_{\gamma}=\sum_{j \geq 0} \gamma_{j} \varphi_{j}$ be such that $v_{\gamma} \in \mathcal{S}^{\prime}\left(\mathbb{R}^{d}\right)$. Denote by $v_{\gamma}^{\omega}=\sum_{j \geq 0} \gamma_{j} X_{j}(\omega) \varphi_{j}$ a randomization of $v_{\gamma}$ with $\nu$ satisfying (1.10).

Recall that $\mathcal{H}_{k}^{s}=\operatorname{Dom}\left(-\Delta+|x|^{2 k}\right)^{\frac{k+1}{2 k} s}$ and $H^{\sigma}=\operatorname{Dom}(-\Delta)^{\frac{\sigma}{2}}$, then

Theorem 3.11. - If for $s \geq 0, v_{\gamma} \notin \mathcal{H}_{k}^{s}\left(\mathbb{R}^{d}\right)$ then a. s. $v_{\gamma}^{\omega} \notin H^{\frac{k+1}{k} s}\left(\mathbb{R}^{d}\right)$ and $v_{\gamma}^{\omega} \notin L^{2}\left(\mathbb{R}^{d},|x|^{(k+1) s} d x\right)$

The first step in the proof is the following

Proposition 3.12. - For every $s \geq 0$ there exist $C_{1}(s)>0, C_{2}(s)>0$ such that

$$
\begin{gathered}
C_{1}(s) \lambda_{n}^{s} \leq\left\|\Delta^{s} \varphi_{n}\right\|_{L^{2}\left(\mathbb{R}^{d}\right)} \leq C_{2}(s) \lambda_{n}^{s}, \quad \forall n \geq 0, \\
C_{1}(s) \lambda_{n}^{s /(2 k)} \leq\left\||x|^{s} \varphi_{n}\right\|_{L^{2}\left(\mathbb{R}^{d}\right)} \leq C_{2}(s) \lambda_{n}^{s /(2 k)}, \forall n \geq 0 .
\end{gathered}
$$

Proof. - By a standard scaling argument, estimate (3.4) is equivalent to the semiclassical estimate

$$
\left.\left.C_{1}(s) \leq\left\|(h \Delta)^{s} \psi_{h}\right\|_{L^{2}\left(\mathbb{R}^{d}\right)} \leq C_{2}(s), \forall h \in\right] 0,1\right],
$$

where $\left(-h^{2} \Delta+V\right) \psi_{h}=\lambda_{h} \psi_{h}$ and $\lim _{h \rightarrow 0} \lambda_{h}=1$.

In (3.4) or (3.6) the upper bound is obvious and we only need to prove the lower bound.

Assume that the lower bound in (3.6) is not satisfied. Let $\mu_{s c}$ be the semiclassical measure of the semiclassical state $\psi_{h}$. It is well known that the support of $\mu_{s c}$ is in the energy surface $H^{-1}(1)$ and is invariant by the classical Hamiltonian flow $\Phi_{H}^{t}$ of $H$. Denote by $A(x, \xi)=|\xi|^{2 s}$. Then for some sequence of $h$ we have

$$
\lim _{h \rightarrow 0}\left\|\hat{A}(h) \psi_{h}\right\|_{L^{2}\left(\mathbb{R}^{d}\right)}=0 .
$$

By definition of the semiclassical measure $\mu_{s c}$, for some subsequence $h_{n}$ we have

$$
\left\langle\hat{A}\left(h_{n}\right) \psi_{h_{n}}, \psi_{h_{n}}\right\rangle \longrightarrow \int_{\mathbb{R}^{2 d}} A(x, \xi) \mathrm{d} \mu_{s c}(x, \xi),
$$

therefore, from (3.7), using that $A \geq 0$ we get that the support of $\mu_{s c}$ is in $A^{-1}(0) \cap H^{-1}(1)$. So if $X_{0}$ is in $\operatorname{supp}\left(\mu_{s c}\right)$ then for every $t \in \mathbb{R}, \Phi_{H}^{t} X_{0} \in \operatorname{supp}\left(\mu_{s c}\right)$. We get a contradiction because $A^{-1}(0)$ cannot contain a global classical trajectory. The same argument applies to the observable $A(x, \xi)=|x|^{s}$, giving (3.5).

Now we prove Theorem 3.11 following $[\mathbf{1 7}$. 
Proof of Theorem [3.11. - Let $\chi$ a smooth cutoff, $0 \leq \chi \leq 1, \operatorname{supp}(\chi) \subseteq[-1,2], \chi=1$ on $[0,1]$. Introduce the notations:

$$
\begin{aligned}
\sigma_{N}^{2} & =\mathbb{E}\left[\left\|\chi\left(\frac{H}{N}\right) v_{\gamma}^{\omega}\right\|_{\mathcal{H}^{s}\left(\mathbb{R}^{d}\right)}^{2}\right]=\sum_{n \geq 1} \chi^{2}\left(\frac{\lambda_{n}}{N}\right)\left|\gamma_{n}\right|^{2} \lambda_{n}^{s} \\
s_{N}(\omega) & =\left\|\chi\left(\frac{H}{N}\right) v_{\gamma}^{\omega}\right\|_{H^{s}\left(\mathbb{R}^{d}\right)} \\
M(\omega) & =\sup _{N \geq 1} s_{N}(\omega) .
\end{aligned}
$$

The event $[M=+\infty]$ is a tail event for the sequence of independent random variables $\left\{X_{n}\right\}_{n \geq 0}$. Thus, according to the (0-1) law, to prove that $\mathbb{P}[M=+\infty]=1$ it is enough to prove that $\mathbb{P}[M=+\infty]>0$. We will use the following Paley-Zygmund inequality: if $X \geq 0$ is a random variable and $\lambda \geq 0$,

$$
\mathbb{P}[X \geq \lambda E(X)] \geq(1-\lambda)^{2} \frac{(E X)^{2}}{E X^{2}} .
$$

Using (3.4) and properties of the sequence $\left\{X_{n}\right\}_{n \geq 0}$ we get easily, for some $c>0, C>0$,

$$
\mathbb{E}\left[s_{N}^{2}\right] \geq c \sigma_{N}^{2} \text { and } \mathbb{E}\left[s_{N}^{4}\right] \leq C \sigma_{N}^{4}, \quad \forall N \geq 1 .
$$

Hence using the Paley-Zygmund inequality, we get

$$
\mathbb{P}\left[M^{2} \geq \frac{c \sigma_{N}^{2}}{2}\right] \geq \frac{c^{2}}{4 C^{2}}, \quad \forall N \geq 1
$$

The result follows from the monotone convergence theorem.

\section{Random quantum ergodicity}

We adapt here in the context of semi-classical Schrödinger operators on $\mathbb{R}^{d}$ results proved for the Laplace operator on compact manifolds [2, Theorem 3].

The spectral results which are necessary in the proof come from [8, and have already been used there to obtain quantum ergodicity results. For an introduction to quantum ergodicity, we refer to Zworski [29, Chapter 15].

4.1. Spectral preparations. - Let us introduce now the assumptions for this section. Let $d \geq 2$ and let $\left.\left.J_{h}=h I_{h}=\right] a_{h}, b_{h}\right]$ be such that

$$
\lim _{h \rightarrow 0} a_{h}=\lim _{h \rightarrow 0} b_{h}=\eta \quad \text { and } \quad \lim _{h \rightarrow 0} \frac{b_{h}-a_{h}}{h}=+\infty .
$$

For a smooth symbol $A(x, \xi)$ in $\mathbb{R}^{2 d}$, we denote by $\hat{A}(h)$ its Weyl $h$-quantization defined by

$$
\hat{A}(h) f(x)=\hat{A}(x, h D) f(x)=\frac{1}{(2 \pi h)^{d}} \int_{\mathbb{R}^{d}} \int_{\mathbb{R}^{d}} e^{i(x-y) \xi / h} A\left(\frac{x+y}{2}, \xi\right) f(y) d y d \xi,
$$

then if $A$ is real, the operator $\hat{A}(h)$ is self-adjoint in $L^{2}\left(\mathbb{R}^{d}\right)$.

Assume that $H(x, \xi)=|\xi|^{2}+V(x)$, with $(x, \xi) \in \mathbb{R}^{2 d}$ where $V$ satisfies the assumptions (A1). Then from [8] (and its bibliography) it is known that: 
(i) In $J_{h}$ the spectrum of $\hat{H}(h)$ is discrete.

(ii) The number $M_{h}$ of eigenvalues of $\hat{H}(h)$ in $J_{h}$ satisfies the Weyl-Hörmander law:

$$
M_{h}=(2 \pi h)^{-d} \int_{\left[(x, \xi) ; H(x, \xi) \in J_{h}\right]} d x d \xi+\mathcal{O}\left(h^{1-d}\right) .
$$

Therefore, thanks to Assumption $(A 1)$ on $V$ we can make the change of variables $y=h^{1 /(k+1)} x$, $\eta=h^{k /(k+1)} \xi$ and get, thanks to (1.7), that $N_{h} \sim M_{h}$ when $h \longrightarrow 0$.

(iii) Denote by $S(1)$ the set of the symbols

$$
S(1)=\left\{A \in C^{\infty}\left(\mathbb{R}^{2 d}\right), \forall \alpha, \forall \beta, \sup _{(x, \xi)}\left|\partial_{x}^{\alpha} \partial_{\xi}^{\beta} A(x, \xi)\right|<+\infty\right\} .
$$

By the Calderon-Vaillancourt theorem, there exist $c>0$ and $\nu_{d} \in \mathbb{N}$ such that for all $A \in S(1)$

$$
\|\hat{A}\|_{L^{2} \rightarrow L^{2}} \leq c\|A\|_{W^{\nu_{d}, \infty}\left(\mathbb{R}^{2 d}\right)} .
$$

Therefore, for later purpose, we introduce

$$
S_{b}(1)=\left\{A \in S(1),\|A\|_{W^{\nu_{d}}, \infty\left(\mathbb{R}^{2 d}\right)} \leq 1\right\} .
$$

Next, let $\Pi_{h}$ be the spectral projector of $\hat{H}(h)$ on $J_{h}$ and $A \in S(1)$, then we have

$$
\operatorname{Tr}\left(\hat{A}(h) \Pi_{h}\right)=(2 \pi h)^{-d} \int_{\left[(x, \xi) ; H(x, \xi) \in J_{h}\right]} A(x, \xi) d x d \xi+\mathcal{O}\left(h^{1-d}\right) .
$$

Denote by $d L_{\eta}$ the Liouville probability measure on the energy surface $H^{-1}(\eta)$, and recall that

$$
d L_{\eta}=C_{\eta} \frac{d \Sigma_{\eta}}{|\nabla H|}
$$

where $d \Sigma_{\eta}$ is the Euclidean measure on $\Sigma_{\eta}:=H^{-1}(\eta)$ and $C_{\eta}$ the normalization constant. Then we can prove that

$$
\frac{\operatorname{Tr}\left(\hat{A}(h) \Pi_{h}\right)}{M_{h}}=L_{\eta}(A)+\mathcal{O}\left(\frac{h^{1-d}}{M_{h}}+b_{h}-a_{h}\right)=L_{\eta}(A)+\mathcal{O}\left(\frac{h}{b_{h}-a_{h}}+b_{h}-a_{h}\right),
$$

so that with our assumptions we have

$$
\lim _{h \rightarrow 0} \frac{\operatorname{Tr}\left(\hat{A}(h) \Pi_{h}\right)}{M_{h}}=L_{\eta}(A) .
$$

As above, $\mathcal{E}_{h}$ is the range of $\Pi_{h}$ and $\mathbf{S}_{h}$ is the (complex) unit sphere of $\mathcal{E}_{h}$, which is of dimension $M_{h}$. In the particular case where $\mathbf{Q}_{h}^{(u)}$ is the uniform probability on $\mathbf{S}_{h}$, using that the canonical probability on the sphere is invariant under the unitary group, we can see easily that the corresponding expectation satisfies

$$
\mathbf{F}_{h}^{(u)}[\langle v, \hat{A}(h) v\rangle]=\frac{\operatorname{Tr}\left(\hat{A}(h) \Pi_{h}\right)}{M_{h}}, \text { for every } v \in \mathbf{S}_{h} .
$$

We shall see later (in (4.7)) that this is still true, up to a small error, for more general probabilities. 
4.2. Proof of Theorem 1.4. - Recall the definition (2.4)

$$
\psi_{j}^{h}(x)=h^{-\frac{d}{2(k+1)}} \varphi_{j}\left(h^{-\frac{1}{k+1}} x\right):=\mathscr{L}_{h} \varphi_{j}(x) .
$$

We define the random vector in $\mathcal{E}_{h}$

$$
\tau(\omega):=\frac{1}{\sqrt{M_{h}}} \sum_{j \in \Lambda_{h}} X_{j}(\omega) \psi_{j}^{(h)}
$$

and we assume that the law of the r.v. $X=\left(X_{j}\right)_{j \geq 1}$ satisfy (1.10). We then consider $\mathbf{Q}_{h}$ the probability on $\mathbf{S}_{h}$ defined by

$$
\int_{\mathbf{S}_{h}} f(u) \mathrm{d} \mathbf{Q}_{h}(u)=\int_{\Omega} f\left(\frac{\tau(\omega)}{\|\tau(\omega)\|_{L^{2}\left(\mathbb{R}^{d}\right)}}\right) \mathrm{d} \mathbb{P}(\omega),
$$

for all measurable and bounded function $f: \mathbf{S}_{h} \longrightarrow \mathbb{R}$. In the sequel we denote by $\mathbf{F}_{h}$ the expectation associated to this probability.

Theorem 1.4 will be implied by the following Proposition, with the unitary transformation $\mathscr{L}_{h}$. Recall the definition (4.2) of $S_{b}(1)$, then

Proposition 4.1. - Under the previous assumptions there exist $c, C>0$ so that for all $r \geq 1$, $h \in] 0,1]$ and $A \in S_{b}(1)$

$$
\mathbf{Q}_{h}\left[u \in \mathbf{S}_{h}:\left|\langle u, \hat{A}(h) u\rangle-L_{\eta}(A)\right|>r\right] \leq C e^{-c M_{h} r^{2}} .
$$

As a consequence, there exists $C>0$ so that for all $p \geq 2, h \in] 0,1]$ and $A \in S(1)$

$$
\left\|\langle u, \hat{A}(h) u\rangle-L_{\eta}(A)\right\|_{L_{\mathbf{Q}_{h}}^{p}} \leq C M_{h}^{-1 / 2} \sqrt{p}
$$

Remark 4.2. - Actually, this result holds true under more general hypotheses on $H$. Namely, it is enough to assume that $H$ is a real smooth symbol on $\mathbb{R}^{2 d}$ so that

$$
\left\{\begin{array}{l}
\bullet\left|\partial_{x}^{\alpha} \partial_{\xi}^{\beta} H(x, \xi)\right| \leq C_{\alpha, \beta}, \quad|\alpha+\beta| \geq m \text { if } m \text { is large enough } \\
\bullet H^{-1}\left[\eta-\varepsilon_{0}, \eta+\varepsilon\right] \text { is compact in } \mathbb{R}^{2 d} \\
\bullet \quad \eta \text { is non critical for } H .
\end{array}\right.
$$

Proof. - Let $A \in S_{b}(1)$. To begin with, we can assume that $\hat{A}(h) \geq 0$, since we can consider the operator $\hat{A}(h)+C$, where $C>0$ is some large constant. Then observe that for all $u, v \in \mathbf{S}_{h}$,

$$
|\langle u, \hat{A}(h) u\rangle-\langle v, \hat{A}(h) v\rangle| \leq 2\|\hat{A}(h)\|_{L^{2} \rightarrow L^{2}}\|u-v\|_{L^{2}\left(\mathbb{R}^{d}\right)} \leq c\|u-v\|_{L^{2}\left(\mathbb{R}^{d}\right)},
$$

thus we can apply [18, Proposition 2.12], which gives $K, \kappa>0$ so that for all $r>0$ and $h \in] 0,1]$

$$
\mathbf{Q}_{h}\left[u \in \mathbf{S}_{h}:|\langle u, \hat{A}(h) u\rangle-\mathcal{M}|>r\right] \leq K \mathrm{e}^{-\kappa M_{h} r^{2}},
$$


where $\mathcal{M}$ is a median for $u \longmapsto\langle u, \hat{A}(h) u\rangle$. Next, by (4.6)

$$
\begin{aligned}
\left|\mathbf{F}_{h}[\langle u, \hat{A}(h) u\rangle]-\mathcal{M}\right| & \leq \mathbf{F}_{h}[|\langle u, \hat{A}(h) u\rangle-\mathcal{M}|] \\
& =\int_{0}^{+\infty} \mathbf{Q}_{h}\left[u \in \mathbf{S}_{h}:|\langle u, \hat{A}(h) u\rangle-\mathcal{M}|>r\right] \mathrm{d} r \leq C M_{h}^{-1 / 2}
\end{aligned}
$$

In order to complete the proof, by (4.3) and the previous lines, it is enough to show that

$$
\mathbf{F}_{h}[\langle u, \hat{A}(h) u\rangle]-\frac{\operatorname{Tr}\left(\hat{A}(h) \Pi_{h}\right)}{M_{h}} \longrightarrow 0,
$$

when $h \longrightarrow 0$. Here the random vector $\tau$ which defines $\mathbf{Q}_{h}$ reads

$$
\tau(\omega)=\frac{1}{\sqrt{M_{h}}} \sum_{j \in \Lambda_{h}} X_{j}(\omega) \psi_{j}^{(h)},
$$

so that

$$
\|\tau(\omega)\|^{2}:=\|\tau(\omega)\|_{L^{2}\left(\mathbb{R}^{d}\right)}^{2}=\frac{1}{M_{h}} \sum_{j=1}^{M_{h}}\left|X_{j}(\omega)\right|^{2} .
$$

By definition

$$
\mathbf{F}_{h}[\langle u, \hat{A}(h) u\rangle]=\int_{\mathbf{S}_{h}}\langle v, \hat{A}(h) v\rangle \mathrm{d} \mathbf{Q}_{h}(v)=\int_{\Omega}\left\langle\frac{\tau(\omega)}{\|\tau(\omega)\|}, \hat{A}(h) \frac{\tau(\omega)}{\|\tau(\omega)\|}\right\rangle \mathrm{d} \mathbb{P}(\omega) .
$$

Denote by

$$
\Omega_{h}=\left\{\omega \in \Omega:\left|\|\tau(\omega)\|_{L^{2}}^{2}-1\right| \leq M_{h}^{-1 / 2}\right\} .
$$

By [18, Lemma 2.11], $\mathbb{P}\left(\Omega_{h}^{c}\right) \leq C \mathrm{e}^{-c M_{h}^{1 / 2}}$. We write

$$
\begin{aligned}
\int_{\Omega}\left\langle\frac{\tau}{\|\tau\|}, \hat{A}(h) \frac{\tau}{\|\tau\|}\right\rangle \mathrm{d} \mathbb{P}(\omega)-\int_{\Omega}\langle\tau, \hat{A}(h) \tau\rangle \mathrm{d} \mathbb{P}(\omega)= \\
\int_{\Omega_{h}} \frac{1-\|\tau\|^{2}}{\|\tau\|^{2}}\langle\tau, \hat{A}(h) \tau\rangle \mathrm{d} \mathbb{P}(\omega)+\int_{\Omega_{h}^{c}} \frac{1-\|\tau\|^{2}}{\|\tau\|^{2}}\langle\tau, \hat{A}(h) \tau\rangle \mathrm{d} \mathbb{P}(\omega):=C_{1, h}+C_{2, h}
\end{aligned}
$$

Firstly, by definition of $\Omega_{h}$ we get $\left|C_{1, h}\right| \leq M_{h}^{-1 / 2}\|\hat{A}(h)\|_{L^{2}}$. Secondly, by Cauchy-Schwarz

$$
\left|C_{2, h}\right| \leq\|\hat{A}(h)\|_{L^{2}} \int_{\Omega_{N}^{c}}\left(1+\|\tau\|^{2}\right) \mathrm{d} \mathbb{P}(\omega) \leq\|\hat{A}(h)\|_{L^{2}} \mathbb{P}^{1 / 2}\left(\Omega_{h}^{c}\right)\left(\int_{\Omega}\left(1+\|\tau\|^{2}\right)^{2} \mathrm{~d} \mathbb{P}(\omega)\right)^{1 / 2}
$$

It remains to check that $\int_{\Omega}\|\tau\|^{4} \mathrm{~d} \mathbb{P}(\omega) \leq C$. By (1.11), there exists $\varepsilon>0$ so that $\int_{\Omega} \mathrm{e}^{2 \varepsilon X_{j}^{2}(\omega)} \mathrm{d} \mathbb{P}(\omega)=$ $C_{\varepsilon}<+\infty$. Thus, by the inequality $\varepsilon^{2}|\tau|^{4} \leq \mathrm{e}^{2 \varepsilon|\tau|^{2}}$ and by Jensen

$$
\varepsilon^{2} \int_{\Omega}\|\tau\|^{4} \mathrm{~d} \mathbb{P}(\omega) \leq \int_{\Omega} \mathrm{e}^{\frac{2 \varepsilon}{M_{h}} \sum_{j=1}^{M_{h}} X_{j}^{2}(\omega)} \mathrm{d} \mathbb{P}(\omega) \leq\left(\int_{\Omega} \mathrm{e}^{2 \varepsilon \sum_{j=1}^{M_{h}} X_{j}^{2}(\omega)} \mathrm{d} \mathbb{P}(\omega)\right)^{1 / M_{h}}=C_{\varepsilon} .
$$

Therefore $C_{1, h}+C_{2, h} \longrightarrow 0$. 
Finally, observe that

$$
\int_{\Omega}\langle\tau, \hat{A}(h) \tau\rangle \mathrm{dP}(\omega)=\frac{1}{M_{h}} \sum_{j=1}^{M_{h}}\left\langle\psi_{j}^{(h)}, \hat{A}(h) \psi_{j}^{(h)}\right\rangle=\frac{\operatorname{Tr}\left(\hat{A}(h) \Pi_{h}\right)}{M_{h}},
$$

and thanks to (4.8), we get (4.7).

Now we can prove Theorem 1.4. To do that we apply Proposition 4.1 to the semiclassical Hamiltonian $\hat{H}_{\varepsilon}(h)=-h^{2} \Delta+V_{\varepsilon}$ depending smoothly on $\varepsilon \in[0,1]$. This reduction is allowed using the following Lemma

Lemma 4.3. - Consider the $\left(\psi_{j}^{h}\right)$ defined by (4.4). Then for every $A \in S(1, k) \subset S(1)$ we have

$$
\left\langle\varphi_{j}, \hat{A} \varphi_{\ell}\right\rangle=\left\langle\psi_{j}^{h}, \hat{A}(h) \psi_{\ell}^{h}\right\rangle+\mathcal{O}\left(h^{\infty}\right)
$$

uniformly in $j, \ell \in \Lambda_{h}$.

Proof. - Let us introduce the unitary transformation $\mathscr{L}_{h} u(x)=h^{-\frac{d}{2(k+1)}} u\left(h^{-\frac{1}{k+1}} x\right)$. We have

$$
\left\langle\varphi_{j}, \hat{A} \varphi_{\ell}\right\rangle=\left\langle\psi_{j}^{h}, \mathscr{L}_{h} \hat{A} \mathscr{L}_{h}^{-1} \psi_{\ell}^{h}\right\rangle
$$

The 1-Weyl symbol $A_{h}$ of $\mathscr{L}_{h} \hat{A} \mathscr{L}_{h}^{-1}$ is $A_{h}(x, \xi)=A\left(h^{-\frac{1}{k+1}} x, h^{\frac{1}{k+1}} \xi\right)$. So its $h$-Weyl symbol is $A(h)(x, \xi)=A\left(h^{-\frac{1}{k+1}} x, h^{-\frac{k}{k+1}} \xi\right)$. Now using that $A$ is quasi-homogeneous outside $(0,0)$, we have for every $h \in] 0,1]$ and $|x|+|\xi| \geq \varepsilon_{0}$, that $A(h)(x, \xi)=A(x, \xi)$.

It is known that, for every $j \in \Lambda_{h}$, the semi-classical wave front set (or the frequency set, see for example [20]) of $\psi_{j}^{h}$ is in a small neighborhood of $H_{0}^{-1}(\tau)$ which do not contain a neighborhood of $(0,0)$ for $\tau$ large enough. Then the Lemma is proved.

Now we easily get the following result, using the Borel-Cantelli Lemma. Assume that the hypothesis of Theorem 1.4 are satisfied.

Let $\left\{h_{j}\right\}_{j \geq 0}$ be a sequence of positive real numbers converging to 0 as $j \rightarrow+\infty$. Define the compact metric space $X=\prod_{j \in \mathbb{N}} \mathbf{S}_{h_{j}}$ equipped with the product probability

$$
\mathbf{P}=\otimes_{j \in \mathbb{N}} \mathbf{P}_{h_{j}} .
$$

Let $u \in X, u=\left\{u_{j}\right\}_{j \in \mathbb{N}}$ where $u_{j} \in \mathbf{S}_{h_{j}}$. For any $A \in S(1, k), u \mapsto\left\langle u_{j}, \hat{A} u_{j}\right\rangle$ defines a sequence of random variables on $X$.

Corollary 4.4. - Assume that $d \geq 2$ and that $\sum_{j \geq 0} \mathrm{e}^{-\varepsilon h_{j}^{1-d}}<+\infty$ for every $\varepsilon>0$. Then

$$
\mathbf{P}\left[u \in X, \lim _{j \rightarrow+\infty}\left\langle u_{j}, \hat{A} u_{j}\right\rangle=L_{\eta}(A), \forall A \in S(1, k)\right]=1 .
$$

Proof. - Denote by $f_{h}(u)=\left\langle\Pi_{h} u, \hat{A} \Pi_{h} u\right\rangle$. Notice that the random variable $f_{h_{j}}$ depends only on $u_{j}=\Pi_{h_{j}} u$ so we get

$$
\mathbf{P}\left[u=\left\{u_{j}\right\}:\left|f_{h_{j}}(u)-L_{\eta}(A)\right| \geq \varepsilon\right]=\mathbf{P}_{h_{j}}\left[\left|f_{h_{j}}-L_{\eta}(A)\right| \geq \varepsilon\right] .
$$


So applying (4.5) and the Borel-Cantelli Lemma to the independent random variables $\left\{f_{h_{j}}\right\}_{j \in \mathbb{N}}$ we get the conclusion.

4.3. Proof of Theorem 1.6. - In (4.1) the condition on $b_{h}-a_{h}$ (the length of the spectral windows) is too restrictive to prove the Theorem (QUE). To achieve the proof we need to relax the condition (4.1), since the harmonic oscillator does not satisfy (4.1). Actually, to isolate its eigenvalues we need to consider spectral windows $\left[a_{h}, a_{h}+2 h[\right.$.

To enlighten the discussion we present a more general setting, inspired from the paper [28] using semi-classical spectral results proved in [7, 16.

Besides the general assumptions on the classical hamiltonian $H$ we shall consider the two following particular cases:

(Per) There exists $\varepsilon_{0}>0$ such that in $H_{0}^{-1}\left[\eta-\varepsilon_{0}, \eta+\varepsilon_{0}\right]$ the hamiltonian flow $\Phi_{H_{0}}^{t}$ defined by $H_{0}$ is periodic with period $T=2 \pi$ and $T$ is a primitive period (there exists no periodic path with period in $] 0, T[)$.

(APer) On the energy shell $\Sigma_{\eta}=H_{0}^{-1}(\eta)$ the set of periodic points is of measure 0 (for the Liouville measure).

For instance, Assumption (Per) is fulfilled with $H_{0}(x, \xi)=\frac{1}{2}\left(|\xi|^{2}+|x|^{2}\right)$ and (Aper) is fulfilled for $d \geq 2$ with $H_{0}(x, \xi)=\frac{1}{2}\left(|\xi|^{2}+\sum_{1 \leq j \leq d} \omega_{j}^{2} x_{j}^{2}\right), \omega_{j}>0$, if $\left(\omega_{1}, \cdots, \omega_{d}\right)$ are independent on $\mathbb{Z}$.

Denote $\sigma(\hat{H})$ the spectrum of $\hat{H}$. Remark that for the isotropic harmonic oscillator the eigenvalues are very degenerate $(d \geq 2)$ and if $\left(\omega_{1}, \cdots, \omega_{d}\right)$ are independent on $\mathbb{Z}$ the eigenvalues are non degenerate.

If condition (Per) is satisfied, it follows from [7] that there exist $\alpha \in \mathbb{Z}, \gamma \in \mathbb{R}, C_{0} \geq 0$ such that

$$
\left.\sigma(\hat{H}) \cap] \eta-\varepsilon_{0}, \eta+\varepsilon_{0}\left[\subseteq \bigcup_{\ell \in \mathbb{Z}}\right] e_{\ell, h}-C_{0} h^{2}, e_{\ell, h}+C_{0} h^{2}\right]
$$

where $e_{\ell, h}=\left(\ell+\frac{\alpha}{4}\right) h+\gamma$.

For the harmonic oscillator we have $\alpha=2$ (Maslov-Morse index), $\gamma=0$ and $C_{0}=0$.

If condition (Aper) is satisfied, it follows from [16] that for every $\delta>0$ there is an infinite number of eigenvalues of $\hat{H}$ in $J_{\delta, h}=\left[a_{h}, a_{h}+\delta h[\right.$. More precisely there exists $C>0$ such that

$$
\#\left\{\sigma(\hat{H}) \cap J_{\delta, h}\right\}=C \delta h^{d-1}+o\left(h^{d-1}\right) .
$$

Our probabilistic results concerning quantum ergodicity are obtained by combining probability technics with the following spectral semi-classical results.

Proposition 4.5. - We have the following asymptotic limit 4.3

$$
\lim _{h \rightarrow 0} \frac{\operatorname{Tr}\left(\hat{A}(h) \Pi_{h}\right)}{M_{h}}=L_{\eta}(A)
$$


for the following choice of the spectral window:

(i) in the "general" case, $J_{h}=\left[a_{h}, a_{h}+h \ell(h)\left[, \lim _{h \rightarrow 0} \ell(h)=+\infty\right.\right.$;

(ii) under assumption (Per), $\left.\left.J_{h}=\right] e_{\ell, h}-C_{0} h^{2}, e_{\ell, h}+C_{0} h^{2}\right]$ such that $\lim _{h \rightarrow 0, \ell \rightarrow+\infty} e_{\ell, h}=\eta$;

(iii) under assumption (Aper), $J_{h}=\left[a_{h}, a_{h}+\delta h\right]$, for any $\delta>0$ where $\lim _{h \rightarrow 0} a_{h}=\eta$.

The previous proposition can be easily deduced from the works [7, 16, 28].

Proof of Theorem 1.6, - We apply Proposition 4.5 to the harmonic oscillator which satisfies the assumption (ii). Then we also have the result of Proposition 4.1 .

Every $B \in \mathcal{B}$ can be identified with $\left\{B_{j}\right\}_{j \geq 1}$ where $B_{j} \in U\left(N_{h_{j}}\right)$. The random variables $D_{j}$ are independent and $D_{j}$ depends only on $B_{j}$. So for every $r>0$ we have

$$
\begin{aligned}
\rho\left[D_{j}(B)>r\right]=\rho_{j}\left[D_{j}(B)>r\right] & =\rho_{j}\left[\exists \ell \in \llbracket 1, N_{h_{j}} \rrbracket,\left|\left\langle\varphi_{j, \ell}, \hat{A} \varphi_{j, \ell}\right\rangle-L_{2}(A)\right|>r\right] \\
& \leq \sum_{1 \leq \ell \leq N_{h_{j}}} \rho_{j}\left[\left|\left\langle\varphi_{j, \ell}, \hat{A} \varphi_{j, \ell}\right\rangle-L_{2}(A)\right|>r\right] \\
& \leq N_{h_{j}} \mathbf{P}_{h_{j}}\left[\left|\left\langle u, \hat{A}\left(h_{j}\right) u\right\rangle-L_{2}(A)\right|>r-C h_{j}^{M}\right] .
\end{aligned}
$$

In the last line we have used Lemma 4.3 where $C \geq 0$ and $M$ arbitrary large, and that the probability $\mathbf{P}_{h}$ is the push-forward of the Haar measure of $U\left(M_{h}\right)$ by the maps: $U\left(N_{h}\right) \ni M \mapsto M v \in \mathbf{S}_{h}$, for any $v \in \mathbf{S}_{h}$.

So using Proposition 4.1 we get, with positive constants $C_{1}, C_{2}, C_{3}$,

$$
\rho\left[D_{j}(B)>r\right] \leq C_{1} j^{d-1} \exp \left[-C_{2} j^{d-1}\left(r-C_{3} j^{-M}\right)^{2}\right] .
$$

In particular for any $d \geq 2$ we get

$$
\sum_{j \geq 1} \rho\left[D_{j}(B)>r\right]<+\infty
$$

and the result is a consequence of the Borel-Cantelli Lemma.

Remark 4.6. - Our proof of unique quantum ergodicity for random bases can be adapted to prove analogous results for the Laplace-Beltrami operator on compact manifolds. Notice that our method is different from the method used in [28, 14]. In particular we do not use the Szegö limit theorem like in [28, prop. 1.2.4] so that we get a slightly better result in the aperiodic case. Let us formulate our result for non isotropic harmonic oscillator.

Let $\hat{H}=\frac{1}{2}\left(-\triangle+\sum_{1 \leq j \leq d} \omega_{j}^{2} x_{j}^{2}\right)$, with $\omega_{j}>0$ and where $\left\{\omega_{1}, \cdots, \omega_{d}\right\}$ are $\mathbb{Z}$-independent.

The eigenvalues of $\hat{H}$ are $\lambda_{\alpha}=\sum_{1 \leq j \leq d}\left(\alpha_{j}+\frac{1}{2}\right) \omega_{j}$. Denote by $I_{\delta, k}=\left[\lambda_{0}+k \delta, \lambda_{0}+(k+1) \delta[, \delta>0\right.$, $k \in \mathbb{N}$. For every $\delta>0$, the number of eigenvalues of $\hat{H}$ in $I_{\delta, k}$ is $N_{\delta, k} \approx k^{d-1}, k \rightarrow+\infty$. Let $\mathcal{E}_{\delta, k}$ be the linear space spanned by the eigenfunctions of $\hat{H}$ with eigenvalues in $I_{\delta, k}$.

Denote by $\mathcal{B}_{\delta}$ the set of orthonormal bases of $L^{2}\left(\mathbb{R}^{d}\right)$ obtained by choosing an orthonormal basis $\mathcal{B}_{\delta}$ 
in each $\mathcal{E}_{\delta, k}$. Like in the proof of Theorem 1.6, $\mathcal{B}_{\delta}$ is equipped with a probability measure $\rho_{\delta}$. Then by applying Proposition 4.5 and Proposition 4.1, $\rho_{\delta}$-almost surely, an orthonormal basis of $L^{2}\left(\mathbb{R}^{d}\right)$ in $\mathcal{B}_{\delta}$ is QUE.

Notice that every $\psi \in \mathcal{E}_{\delta, k}$ is a $\delta$-quasimode for $\hat{H}$ :

$$
\hat{H} \psi=\left(\lambda_{0}+k \delta\right) \psi+O(\delta)\|\psi\|_{L^{2}\left(\mathbb{R}^{d}\right)} .
$$

Remark 4.7. - For Schrödinger operators with super-quadratic potentials we could also get a similar result, considering orthonormal basis of quasi-modes (approximated eigenfunctions), using Proposition 4.1 and taking the appropriate power of $-\triangle+V$.

Remark 4.8. - We have supposed that the observables $A$ are of order 0. More generally, we say that $A$ is of order $m, m>0$, if $A$ is an asymptotic sum of quasi-homogeneous symbols of degree $\leq m$ (for more details see [4, 19]). We say that $A$ is quasi-homogeneous of degree $m \in \mathbb{R}$ if $A\left(r x, r^{k} \xi\right)=$ $r^{(k+1) m} A(x, \xi)$ for $r \geq 1,|(x, \xi)|>\varepsilon$. So if $A$ quasi-homogeneous of degree $m$ then $H_{n o r}^{-m / 2} A H_{n o r}^{-m / 2}$ is quasi-homogeneous of degree 0 . So the previous results holds true for $\frac{\left\langle\varphi_{j}, \hat{A} \varphi_{j}\right\rangle}{\omega_{j}^{m}}$, where $\omega_{j}=\lambda_{j}^{(k+1) / 2 k}$.

\section{Appendix A}

\section{Some point-wise spectral estimates for confining potentials}

The aim of this appendix is to prove Proposition 2.1. Proposition 2.5 and Lemma3.7 for $\delta<2 / 3$. We shall restrict here our analysis to Schrödinger Hamiltonians with polynomial potentials, for simplicity. We shall use a global pseudo-differential calculus with a diagonal metric $g=\frac{d x+d \xi}{w(x, \xi)}$ where $w$ is a weight function on the phase space $\mathbb{R}_{x}^{d} \times \mathbb{R}_{\xi}^{d}$. The general theory was achieved by L. Hörmander with the Weyl-Wigner ordering calculus [9] after C. Fefferman and R. Beals for the "usual" ordering. The construction of parametrices for resolvent of elliptic operators is well known. But to cope with $\delta<2 / 3$ we need to take care of remainder estimates.

A.1. Parametrix for the resolvent. - We assume that the potential $V$ is an elliptic polynomial of degree $2 k$, which means that $C_{1}\langle x\rangle^{2 k} \leq V(x) \leq C_{2}\langle x\rangle^{2 k}$ for $|x|>R$ where $C>0, R>0$. Denote by

$$
\hat{H}(h)=-h^{2} \Delta+V
$$

and $H(x, \xi)=|\xi|^{2}+V(x)$ its semi-classical symbol. We can get accurate approximations for the resolvent $(\hat{H}(h)-z)^{-1}$ for $h>0$ small and $z \in \mathbb{C}$. It is not difficult to get a formal asymptotics as a series in $h$ but to get spectral estimates we need to control the remainder terms when $|z|$ is large (see [20, 5]). Recall that the $h$-Weyl quantization of a smooth symbol $A$ in $\mathbb{R}_{x}^{d} \times \mathbb{R}_{\xi}^{d}$ is

$$
O p_{h}(A) \psi(x)=\hat{A}(h) \psi(x)=(2 \pi h)^{-d} \iint_{\mathbb{R}^{2 d}} A\left(\frac{x+y}{2}, \xi\right) \mathrm{e}^{i h^{-1}(x-y) \cdot \xi} \psi(y) d y d \xi
$$


In particular the Schwartz kernel $K_{A, h}(x, y)$ of $\hat{A}(h)$ is given by

$$
K_{A, h}(x, y)=(2 \pi h)^{-d} \int_{\mathbb{R}^{d}} A\left(\frac{x+y}{2}, \xi\right) \mathrm{e}^{i h^{-1}(x-y) \cdot \xi} d \xi
$$

The basic formula for the symbolic computation is the Moyal product formula. Let $A, B$ be two smooth observables (for example in the Schwartz space $\mathcal{S}\left(\mathbb{R}^{d} \times \mathbb{R}^{d}\right.$ ) and $C$ the $h$-Weyl symbol of the operator product $\hat{C}(h):=\hat{A}(h) \hat{B}(h)$. Then the $h$-Weyl symbol of $\hat{C}(h)$ is a smooth function $C(h, x, \xi)$ given by

$$
C(h ; x, \xi)=\left.\exp \left(\frac{i h}{2} \sigma\left(D_{x}, D_{\xi} ; D_{y}, D_{\eta}\right)\right) A(x, \xi) B(y, \eta)\right|_{(x, \xi)=(y, \eta)},
$$

where $\sigma$ is the standard symplectic form in $\mathbb{R}^{d} \times \mathbb{R}^{d}, \sigma(x, \xi ; x y, \eta)=x \cdot \eta-\xi \cdot y$.

In semiclassical analysis, it is useful to expand the exponent in (A.1), so we get the formal series in $h$ :

$$
\begin{array}{r}
C(h ; x, \xi)=\sum_{j \geq 0} C_{j}(x, \xi) h^{j}, \text { where } \\
C_{j}(x, \xi)=\left.\frac{1}{j !}\left(\frac{i}{2} \sigma\left(D_{x}, D_{\xi} ; D_{y}, D_{\eta}\right)\right)^{j} A(x, \xi) B(y, \eta)\right|_{(x, \xi)=(y, \eta)} .
\end{array}
$$

The crucial point here is to have good estimates for the error term not only in $h$ but also in some extra spectral parameters as we shall see later

$$
R_{N}(h ; x, \xi)=C(h ; x, \xi)-\sum_{0 \leq j \leq N} h^{j} C_{j}(x, \xi)
$$

Here one of the symbols $A, B$ is a polynomial in $(x, \xi)$ so the analysis is much simpler. Let us introduce the class of symbols $\Sigma(r), r \in \mathbb{R}$. For our application here it is enough to consider the weight function

$$
\mu(x, \xi)=\left(1+|x|^{2 k}+|\xi|^{2}\right)^{1 / 2 k}
$$

(a more general setting is considered in $[\mathbf{1 9}, \mathbf{5}$ ). The condition $A \in \Sigma(r)$ means that for every $j \in \mathbb{N}$ we have

$$
s_{j}(A, r):=\sup _{|\alpha+\beta|=j,(x, \xi) \in \mathbb{R}^{2 d}} \mu(x, \xi)^{-r+j}\left|\partial_{\xi}^{\alpha} \partial_{x}^{\beta} A(x, \xi)\right|<+\infty .
$$

The topology on $\Sigma(r)$ is defined by the semi-norms $s_{j}(\cdot, r)$. A basic result concerning Weyl quantization is that for every $h>0,(A, B) \mapsto A \# B$ is continuous for $\Sigma(r) \times \Sigma(s)$ into $\Sigma(r+s)$. In [5, Appendix B], we can find accurate estimates for $R_{N}(h ; x, \xi)$ in a more general setting. Here, using that $\hat{H}(h)$ is a polynomial in $(x, \xi)$ we can perform more explicit computations to construct a parametrix for $(\hat{H}(h)-z)^{-1}$. 
Following [20, p. 134] we construct a parametrix as follows. By induction on $j \in \mathbb{N}$ we define

$$
\begin{aligned}
b_{z, 0} & =(H-z)^{-1} \\
b_{z, j+1} & =-b_{z, 0}\left(\sum_{\substack{\ell+|\alpha+\beta|=j+1 \\
0 \leq \ell \leq j}} \nu(\alpha, \beta)\left(\partial_{\xi}^{\alpha} \partial_{x}^{\beta} H\right)\left(\partial_{\xi}^{\beta} \partial_{x}^{\alpha} b_{z, \ell}\right)\right) \\
B_{z, N}(h) & =\sum_{0 \leq j \leq N} h^{j} b_{z, j},
\end{aligned}
$$

where $\nu(\alpha, \beta)=(-1)^{|\alpha|}\left[\alpha ! \beta ! 2^{|\alpha+\beta|}\right]^{-1}$. Then we have

$$
\hat{B}_{z, N}(h)(\hat{H}(h)-z)=1+\hat{E}_{z, N}(h),
$$

and for our purpose we have to estimate the error term symbol

$$
E_{z, N}(h)=B_{z, N}(h) \#(H-z)-1 .
$$

This Moyal product has a finite expansion in $h$ because the symbol $H$ is a polynomial in $(x, \xi)$. Let us recall that the symbols $b_{z, j}$ have the following properties (see [5])

$$
b_{z, j}=\sum_{0 \leq \ell \leq 2 j-1}(-1)^{\ell} d_{j \ell}(H-z)^{-\ell-1}, j \geq 1
$$

where $d_{j \ell}$ are polynomials in $\partial_{\xi}^{\alpha} \partial_{x}^{\beta} H$ for $1 \leq|\alpha+\beta| \leq j$. Moreover, for all $m \in \mathbb{N}$ we have if $j=2 m$ then $d_{j \ell}=0$ for every $\ell \geq 3 m$ and if $j=2 m+1$ then $d_{j \ell}=0$ for every $\ell \geq 3 m+1$. Furthermore $d_{j \ell}$ is in the symbol class $\Sigma(2 k \ell-2 j)$. Thanks to this vanishing property, we get with the Calderon-Vaillancourt theorem, that there exists $N_{0} \geq 1$ such that for $N \geq 1$

$$
\left\|\hat{B}_{z, N}(h)\right\|_{\mathcal{L}\left(L^{2}, L^{2}\right)} \leq C_{N}\left(1+h^{N}|\operatorname{Im} z|^{-3 N / 2}\right)|\operatorname{Im} z|^{-N_{0}} .
$$

Next, with (A.2) we get the following estimate: There exists $n_{0}$ such that for every $N \geq 1,(\alpha, \beta) \in \mathbb{N}^{2 d}$, there exists $C>0$ such that for every $h \in] 0,1]$ and $z \in \mathbb{C}_{\gamma_{0}}$, we have

$$
\left|\partial_{\xi}^{\alpha} \partial_{x}^{\beta} E_{z, N}(h)\right| \leq C h^{N+1} \sum_{\frac{N+1}{2 k} \leq \ell \leq 3 N / 2+n_{0}+|\alpha+\beta|}\left|\frac{H}{H-z}\right|^{\ell+1}(\mu(x, \xi))^{-2(N+1)-|(\alpha+\beta)|} .
$$

In the r.h.s of A.5 it is desirable to have large decay in $|z|$ and in $\mu$. First, it is known that there exists $\gamma_{0} \in \mathbb{R}$ such that the spectrum of $\hat{H}(h)$ is in $\left[\gamma_{0},+\infty\right.$ [ for all $\left.\left.h \in\right] 0,1\right]$. Then, with elementary considerations, we have that for every $\theta \in[0,1]$

$$
\left|\frac{H}{H-z}\right| \leq \frac{1}{|\sin \varphi|}|z|^{-\theta} H^{\theta} \leq \frac{1}{|\sin \varphi|}|z|^{-\theta} \mu^{2 k \theta},
$$

where $\varphi=\arg z, 0<|\varphi| \leq \pi / 2,|z| \geq \gamma_{0} / 2$.

So we can choose $\theta \in] 0,1\left[\right.$ such that there exist positive numbers $\delta_{1}, \delta_{2}, \delta_{3}, \delta_{4}>0, N_{0}>0$ and $C>0$ such that for $|z| \geq \gamma_{0} / 2,0<|\varphi| \leq \pi / 2,(x, \xi) \in \mathbb{R}^{2 d}, N \geq 1$, we have

$$
\left|\partial_{\xi}^{\alpha} \partial_{x}^{\beta} E_{z, N}(h)\right| \leq C h^{N+1}|\sin \varphi|^{-3 N / 2-N_{0}-|\alpha+\beta|}|z|^{-\delta_{1} N-\delta_{2}|\alpha+\beta|} \mu^{-\delta_{3} N-\delta_{4}|\alpha+\beta|} .
$$


As a result, by the Calderon-Vaillancourt theorem, we obtain that $\hat{E}_{z, N}(h)$ is continuous from $L^{2}\left(\mathbb{R}^{d}\right)$ into $\mathcal{H}_{k}^{\delta N}$ for some $\delta>0$ and that there exist $N_{0} \geq 1, \kappa>0, C>0$ such that for $N \geq 1$

$$
\left\|\hat{E}_{z, N}(h)\right\|_{\mathcal{L}\left(L^{2}, \mathcal{H}_{k}^{\delta N}\right)} \leq C h^{N+1}|\sin \varphi|^{-3 N / 2-N_{0}}|z|^{-\kappa N} .
$$

Since we work with the Weyl quantization, we can get a right parametrix by taking the adjoint of (A.3), and therefore

$$
(\hat{H}(h)-z) \hat{B}_{\bar{z}, N}^{*}(h)=1+\hat{E}_{\bar{z}, N}^{*}(h),
$$

which combined with (A.3) yields

$$
\hat{B}_{z, N}(h)-(\hat{H}(h)-z)^{-1}=\hat{E}_{z, N}(h) \hat{B}_{\bar{z}, N}^{*}(h)-\hat{E}_{z, N}(h)(\hat{H}(h)-z)^{-1} \hat{E}_{\bar{z}, N}^{*}(h):=\hat{R}_{z, N}(h) .
$$

So we can easily see that $\hat{R}_{z, N}(h)$ is a smoothing operator in the weighted Sobolev scale spaces $\left\{\mathcal{H}_{k}^{s}\right\}_{s \in \mathbb{R}}$ when $N$ is large, and we can use the next lemma proved in [19, Proposition 1.3] to give an estimate of the kernel of $\hat{R}_{z, N}(h)$.

Lemma A.1. - Let $\hat{R}, \hat{S}$ be bounded operators in $L^{2}\left(\mathbb{R}^{d}\right)$ and assume that $\hat{R}\left(L^{2}\left(\mathbb{R}^{d}\right)\right) \subseteq \mathcal{H}_{k}^{m}$ and $\hat{S}\left(L^{2}\left(\mathbb{R}^{d}\right)\right) \subseteq \mathcal{H}_{k}^{m}$ with $m>\frac{k d}{k+1}$. Then the operator $\hat{T}:=\hat{R} \hat{S}^{*}$ has a (Lipschitz) continuous Schwartz kernel $K_{T}(x, y)$ on $\mathbb{R}^{d} \times \mathbb{R}^{d}$ and we have the estimate

$$
\left|K_{T}(x, y)\right| \leq C_{k, d, m} W_{m}(x) W_{m}(y)\|\hat{R}\|_{\mathcal{L}\left(L^{2}, \mathcal{H}_{k}^{m}\right)}\|\hat{S}\|_{\mathcal{L}\left(L^{2}, \mathcal{H}_{k}^{m}\right)}
$$

where $C_{k, d, m}$ only depends on $k, d, m$ and $W_{m}(x)=\left(1+|x|^{2 k}\right)^{d / 2-(k+1) m /(2 k)}$.

Remark A.2. - If $m$ can be taken larger then we get that $K_{T}$ has derivatives in $(x, y)$ with corresponding estimates.

Using (A.6), (A.8), (A.9), we get the following estimate for the error term in the parametrix. Let us denote by $K_{R_{z, N}(h)}$ the Schwartz kernel of $\hat{R}_{z, N}(h)$.

Lemma A.3. - There exist $N_{0}$ large enough and $\kappa_{1}, \kappa_{2}>0, C>0$ such that for $N \geq 1$

$$
K_{R_{z, N}(h)}(x, y) \leq C h^{N+1}\left(1+h^{N}|\sin \varphi|^{-3 N / 2}\right)|\sin \varphi|^{-3 N / 2-N_{0}}|z|^{-\kappa_{1} N}\left(1+|x|^{2 k}+|y|^{2 k}\right)^{-\kappa_{2} N}
$$

for all $h \in] 0,1],|z| \geq \gamma_{0} / 2$ with $\arg z=\varphi$ and $x, y \in \mathbb{R}^{d}$.

Moreover, if $z \in \mathbb{C} \backslash \mathbb{R}$ is such that $|z| \leq A$,

$$
K_{R_{z, N}(h)}(x, y) \leq C h^{N+1}\left(1+h^{N}|\operatorname{Im} z|^{-3 N / 2}\right)|\operatorname{Im} z|^{-3 N / 2-N_{0}}\left(1+|x|^{2 k}+|y|^{2 k}\right)^{-\kappa_{2} N} .
$$

Proof. - The estimate of (A.11) is a direct consequence of (A.10) by writing $|\sin \varphi| \geq|\operatorname{Im} z| / A$. For (A.10), we use Lemma A.1 twice, using the estimates (A.4) and (A.7). We write $\hat{E}_{z, N}(h) \hat{B}_{\bar{z}, N}^{*}(h)=$ $\hat{R} \hat{S}^{*}$ with $\hat{R}=\hat{E}_{z, N}(h)(\hat{H}(h)-z)^{\eta N}$ and $\hat{S}=\hat{B}_{\bar{z}, N}(h)(\hat{H}(h)-\bar{z})^{-\eta N}$, with $\eta>0$ small enough so that the hypothesis of Lemma A.1 is satisfied. For the second term, we write $E_{z, N}(h)(\hat{H}(h)-z)^{-1} \hat{E}_{\bar{z}, N}^{*}(h)=\hat{R} \hat{S}^{*}$ with $\hat{R}=\hat{E}_{z, N}(h)$ and $\hat{S}=\hat{E}_{\bar{z}, N}(h)(\hat{H}(h)-\bar{z})^{-1}$. 


\section{A.2. Proof of Proposition 2.1. -}

Proof. - We use the previous result with $h=1$, and we denote by $\hat{H}=\hat{H}(1)$. The heat operator $\mathrm{e}^{-t \hat{H}}$ is related with the resolvent by a Cauchy integral

$$
\mathrm{e}^{-t \hat{H}}=\frac{1}{2 i \pi} \oint_{\Gamma} \mathrm{e}^{-t z}(\hat{H}-z)^{-1} d z
$$

where $\Gamma$ is the following contour in the complex plane. Fix $\varphi \in] 0, \pi / 2]$. Let $z_{0}=\left(\gamma_{0} / 2\right) \mathrm{e}^{i \varphi}$. Let $\Gamma_{+}$ be the line $\left[z_{0}+r \mathrm{e}^{i \varphi}, r \geq 0\right], \Gamma_{-}=\overline{\Gamma_{+}}, \Gamma_{0}=\left[\left(\gamma_{0} / 2\right) \mathrm{e}^{i \psi},-\varphi \leq \psi \leq \varphi\right]$. So $\Gamma=\Gamma_{0} \cup \Gamma_{-} \cup \Gamma_{+}$with a suitable orientation.

We give the main steps of the end of the proof.

- Thanks to the parametrix computed for $(\hat{H}-z)^{-1}$, we have

$$
\mathrm{e}^{-t \hat{H}}=\frac{1}{2 i \pi} O p_{1}\left(\sum_{j=1}^{2 N} f_{j}(x, \xi) \oint_{\Gamma} \mathrm{e}^{-t z}(H(x, \xi)-z)^{-j} d z\right)-\frac{1}{2 i \pi} \oint_{\Gamma} \mathrm{e}^{-t z} \hat{R}_{z, N}(1) d z,
$$

where the $f_{j}$ are linear combinations of the $d_{j \ell}$, hence they are polynomials in $(x, \xi)$.

- The kernel of the main contribution is obtained with the residue theorem:

$$
\frac{1}{2 i \pi} \oint_{\Gamma} \mathrm{e}^{-t z}(H(x, \xi)-z)^{-j} d z=\frac{t^{j-1}}{(j-1) !} \mathrm{e}^{-t H(x, \xi)},
$$

and using degree considerations of the $f_{j}$.

- For $N \geq 1$ large enough, the kernel of the remainder term is estimated with (A.10).

\section{A.3. Proof of Proposition 2.5, -}

Proof. - We use here the semi-classical functional calculus for smooth functions with compact support, and we apply it to the operator $\hat{H}(h)=-h^{2} \Delta+V_{\varepsilon}$ where $V_{\varepsilon}(x)=\varepsilon^{2 k} V(x / \varepsilon)$. We can check that all the previous estimates hold true uniformly in $\varepsilon>0$.

Let $f$ be a non negative $C^{\infty}$ function in ] $-2 C_{0}, 2 C_{0}$ [ with a compact support, such that $f=1$ in $\left[-C_{0}, C_{0}\right]$. Using the spectral theorem for general self-adjoint operators, we can consider the new operator

$$
g(\hat{H}(h))=f\left(\frac{\hat{H}(h)-\nu}{h^{\delta}}\right) .
$$

The operator $g(\hat{H}(h))$ has clearly a smooth Schwartz kernel $K_{f, h}$ (compute it in a basis of eigenvector of $\hat{H}(h))$ and we have

$$
\left|\pi_{H(h)}(\nu+\mu h ; x, x)-\pi_{H(h)}(\nu ; x, x)\right| \leq K_{f, h}(x, x), \quad \forall x \in \mathbb{R}^{d},
$$

where $|\mu| \leq C h^{\delta-1}$. So it is enough to show that $K_{f, h}(x, x)=\mathcal{O}\left(h^{\delta-d}\right)$ uniformly in $x \in \mathbb{R}^{d}$. 
Let us recall the almost-analytic formula for the functional calculus (see [6, Chapter 8])

$$
g(\hat{H}(h))=\frac{1}{\pi} \int_{\mathbb{C}} \bar{\partial} \tilde{g}(z)(\hat{H}(h)-z)^{-1} L(d z),
$$

where $L(d z)=d x d y$ is the Lebesgue measure and where $\tilde{g}$ is an almost analytic extension of $g$. This means that $g$ is $C^{\infty}$ in $\mathbb{C}$ and satisfies, for all $M \geq 0$,

$$
|\bar{\partial} \tilde{g}(z)| \leq C_{M}|\operatorname{Im} z|^{M}, \quad \forall z \in \mathbb{C} .
$$

We can choose $\tilde{g}$ supported in a small complex neighborhood of ] $-2 C_{0}, 2 C_{0}[$. Moreover it results from [6] that $C_{M}$ is estimated by some semi-norms of $g$.

$$
|\bar{\partial} \tilde{g}(z)| \leq \gamma_{M}|\operatorname{Im} z|^{M}\left(\left\|\widehat{D_{x}^{M+1}} g\right\|_{L^{1}}+\left\|D_{x}^{M+2} g\right\|_{L^{1}}\right), \quad \forall z \in \mathbb{C} .
$$

We give the main steps of the end of the proof.

- We plug the parametrix of $(\hat{H}(h)-z)^{-1}$ in (A.13) to compute the kernel of $g(\hat{H}(h))$.

- The kernel of the main contribution $\hat{B}_{z, N}$ is computed explicitly. Actually, the first term in the expansion of $g(\hat{H}(h))$ is

$$
O p_{h}\left(\frac{1}{\pi} \int_{\mathbb{C}} \bar{\partial} \tilde{g}(z)(H-z)^{-1} L(d z)\right)=O p_{h}(g(H))=O p_{h}\left(f\left(\frac{H-\nu}{h^{\delta}}\right)\right),
$$

where we used that $\frac{1}{\pi z}$ is a fundamental solution of $\bar{\partial}$. Therefore, the principal term of $K_{f, h}$ is given by the following formula

$$
K_{f, h}^{0}(x, x)=(2 \pi h)^{-d} \int_{\mathbb{R}^{d}} f\left(\frac{H(x, \xi)-\nu}{h^{\delta}}\right) d \xi,
$$

which implies $K_{f, h}^{0}(x, x)=\mathcal{O}\left(h^{\delta-d}\right)$.

- Choosing $M$ large enough we can estimate the contribution of $\hat{R}_{z, N}(h)$ by using (A.11) and (A.14) for the smallest integer $M \geq 3 N+N_{0}$. In (A.14) the loss in $h$ is $h^{-\delta M}$ but this is compensated by the factor $h^{N+1}$ for $N$ large if we choose $\delta<2 / 3$.

\section{A.4. Proof of Lemma 3.7, -}

Proof. - The proof uses the same tools as for the proof of Proposition 2.5. We choose two cutoff functions $f_{ \pm}$with $f_{+}$as above and $f_{-}$such that $\left.\operatorname{supp}\left(f_{-}\right) \subseteq\right] C_{1}, C_{0}\left[, f_{-}=1\right.$ in $\left[2 C_{1}, C_{0} / 2\right]$ where $C_{1}<C_{0} / 4$. If $K_{f, h}$ is the Schwartz kernel of $g(\hat{H}(h))$ given par A.12 we have

$$
K_{f_{-}, h} \leq e_{x, h} \leq K_{f_{+}, h}(x) .
$$

So we have to prove

$$
C_{0} N_{h} h^{\beta_{2 p, \theta}} \leq\left(\int_{\mathbb{R}^{d}}\langle x\rangle^{k \theta(p-1)} K_{f, h}^{p}(x) d x\right)^{1 / p} \leq C_{1} N_{h} h^{\beta_{2 p, \theta}}
$$

for every $f$ like in A.12). 
We use again the almost-analytic functional calculus. From (A.10) we see that the contribution of the error term of the resolvent parametrix is of order $\mathcal{O}\left(h^{N+1}\right)$. As previously, the principal term of $K_{f, h}$ is given by (A.15), hence we get (A.16) from a two side estimate of this integral.

A.5. On the case $\delta=1$. - Here we show how Proposition 2.5 for $\delta=1$ can be deduced directly from results established in [12]. We admit here that these results can be extended for $V$ depending on a parameter $\varepsilon$.

- Estimates outside the turning points: Outside the turning points $V(x)=\nu$ the estimate can be proved using a standard WKB approximation for the propagator $U(t):=\mathrm{e}^{-i t h^{-1} \hat{H}(h)}$. Let $U(t, x, y)$ be the Schwartz kernel of $U(t)$. Let us give here a sketch of the proof.

Let $\rho \in C_{0}^{\infty}(]-T_{0}, T_{0}[)$ with $T_{0}>0$ small enough. We have

$$
I_{x, \nu}(h):=\int \rho(t) \mathrm{e}^{i t \nu h^{-1}} U(t, x, x) d t=\sum_{j \geq 0} \hat{\rho}\left(\frac{\lambda_{j}-\nu}{h}\right)\left|\varphi_{j}(x)\right|^{2} .
$$

Choosing $\rho$ even such that $\hat{\rho} \geq 0$ and $\hat{\rho}(0)=1$ it is enough to prove

$$
I_{x, \nu}(h)=\mathcal{O}\left(h^{1-d}\right) .
$$

We consider now a WKB approximation for $I_{x, \nu}(h)$

$$
I_{x, \nu}(h) \approx(2 \pi h)^{-d} \int_{\mathbb{R}} \int_{\mathbb{R}^{d}} \rho(t) \mathrm{e}^{i h^{-1}(S(t, x, \eta)-y \cdot \eta+t \nu)}\left(\sum_{j \geq 0} h^{j} a_{j}(t, x, \eta)\right) d t d \eta .
$$

Using a localization energy argument it is enough to consider a bounded open set of the phase space $\left.\mathcal{V}=H^{-1}\right] \nu_{0}-\varepsilon_{0}, \nu_{0}+\varepsilon_{0}[$.

The term $S(t, x, \eta)$ is the solution of the Hamilton-Jacobi equation in $\mathcal{V}$ and the $a_{j}$ are determined by transport equations (see [20] for details).

The stationary points of the phase $\Phi_{x, \nu}(t, \eta):=S(t, x, \eta)-y \cdot \eta+t \nu$ satisfy the equations $t=0$, $|\eta|^{2}=\nu-V(x)$ if $T_{0}$ is chosen small enough. So if $V(x)<\nu$ the critical set is a smooth submanifold $\mathcal{C}_{x, \nu}$ of $\mathbb{R} \times \mathbb{R}^{d}$ of codimension 2 and the Hessian of $\Phi_{x, \nu}$ is non degenerate in the normal directions to $\mathcal{C}_{x, \nu}$. So the stationary phase theorem gives that $I_{x, \nu}(h)=\mathcal{O}\left(h^{1-d}\right)$.

If $V(x)>\nu$ the non-stationary phase theorem gives that $I_{x, \nu}(h)=\mathcal{O}\left(h^{\infty}\right)$.

- Estimates at the turning points: Now we consider the case $|V(x)-\nu|<\varepsilon_{1}$ for $\varepsilon_{1}>0$ arbitrary small. We give the argument used in [12, which is completely different.

Let $\Pi_{h}$ the spectral projector for $\hat{H}(h)$ on $[\nu, \nu+\mu h]$. We claim that it is enough to prove

$$
\left\|\Pi_{h}\right\|_{L^{2} \rightarrow L^{\infty}}=\mathcal{O}\left(h^{\frac{1-d}{2}}\right) .
$$

Assume that A.18 holds true. Denote by $e_{h}(x, y)$ the Schwartz kernel of the projector $\Pi_{h}$ and set $u_{x}: y \mapsto e_{h}(x, y)$. Then we have $\Pi_{h} u_{x}=u_{x}$, thus

$$
e_{h}(x, x)=u_{x}(x)=\left(\Pi_{h} u_{x}\right)(x) \leq\left\|\Pi_{h}\right\|_{L^{2} \rightarrow L^{\infty}}\left\|u_{x}\right\|_{L^{2}} .
$$


Then using that $\Pi_{h}^{2}=\Pi_{h}$ we get

$$
\left\|u_{x}\right\|_{L^{2}}^{2}=\int\left|e_{h}(x, y)\right|^{2} d y=e_{h}(x, x)
$$

which entails that $e_{h}(x, x)=\mathcal{O}\left(h^{1-d}\right)$, thanks to (A.18).

Let $\chi$ be supported in $\mathcal{V} \cap\left\{|V(x)-\nu|<\varepsilon_{1}\right\}$. It is enough to prove that

$$
\left\|\hat{\chi}(h) \Pi_{h}\right\|_{L^{2} \rightarrow L^{\infty}}=\mathcal{O}\left(h^{\frac{1-d}{2}}\right) .
$$

Using that $\left\|(\hat{H}(h)-\nu) \Pi_{h}\right\|_{L^{2} \rightarrow L^{2}}=\mathcal{O}(h)$, for $d \geq 3$ estimate (A.19) is a direct consequence of [12, Theorem 6]. For $d=2$ this theorem gives the estimate $\mathcal{O}\left(h^{-1 / 2}|\log h|^{1 / 2}\right)$, but applying the more difficult [12, Theorem 6], the log term can be eliminated.

\section{References}

[1] N. Burq. Mesures semi-classiques et mesures de défaut (d'après P.Gérard, L.Tartar et al.). Astérisque 245, p. 167-196, Séminaire Bourbaki 1996-1997.

[2] N. Burq and G. Lebeau. Injections de Sobolev probabilistes et applications. Ann. Sci. Éc. Norm. Supér., 46, fascicule 6 (2013), p. 917-962.

[3] Y. Colin de Verdière. Ergodicité et fonctions propres du Laplacien Comm. in Math. Phys., 102, 497-502, (1985).

[4] B. Helffer and D. Robert. Propriétés asymptotiques du spectre d'opérateurs pseudodifférentiels sur $\mathbb{R}^{n}$. Comm. in P.D.E. 7(7), p. 795-882, (1982).

[5] M. Dauge and D. Robert. Weyl's formula for a class of pseudodifferential operators with negative order on $L^{2}\left(\mathbb{R}^{n}\right)$, Lecture Notes in Math. No. 1259, 90-122, Springer-Verlag (1986).

[6] M. Dimassi and J. Sjöstrand. Spectral asymptotics in the Semi-Classical Limit. Cambridge University Press (1999).

[7] B. Helffer and D. Robert Puits de potentiels généralisés et asymptotique semi-classique. Annales de l' I. H. P., section A, tome 41, no. 3, (1984),291-331.

[8] B. Helffer, A. Martinez and D. Robert. Ergodicité et limite semi-classique. Comm. Math. Phys. 109 (1987) $313-326$.

[9] L. Hörmander. The Weyl calculus of pseudodifferential operators. C.P.A.M. 32, p. 359-443, (1979).

[10] V. Ivrii. Microlocal Analysis and Precise Spectral Asymptotics. Springer Monographs in Mathematics (1998).

[11] G.E. Karadzhov. Riesz summability of multiple Hermite series in $L^{p}$ spaces. Math. Z. 219, p. 107-118, (1995).

[12] H. Koch, D. Tataru and M. Zworski. Semiclassical L $L^{p}$ Estimates. Annales Henri Poincaré 8 (2007), 885916.

[13] M. Ledoux. The concentration of measure phenomenon. Mathematical Surveys and Monographs, Vol.89, AMS, (2001).

[14] K. Maples. Quantum unique ergodicity for random bases of spectral projections. Math. Res. Lett. 20 (2013), no. $6,1115-1124$.

[15] A. Mohamed. Comportement asymptotique, avec estimation du reste, des valeurs propres d'une classe d'opérateurs pseudo-différentiels sur $\mathbb{R}^{n}$. Math. Nachr. 140 (1989), 127-186.

[16] V. Petkov and D. Robert Asymptotique semi-classique du spectre d'hamiltoniens quantiques et trajectoires classiques périodiques Comm in P. D. E(1985) 10, 365-390. 
[17] A. Poiret. Solutions globales pour des équations de Schrödinger sur-critiques en toutes dimensions. Preprint : arXiv:120\%.3519.

[18] A. Poiret, D. Robert and L. Thomann. Random weighted Sobolev inequalities on $\mathbb{R}^{d}$ and application to Hermite functions. Ann. Henri Poincaré. 16 (2015), no. 2, 651-689.

[19] D. Robert. Propriétés spectrales d'opérateurs pseudodifférentiels, Comm. in P.D.E. 3 (9) p. 755-826 (1978).

[20] D. Robert. Autour de l'approximation semi-classique. Birkhäuser Boston, (1987).

[21] A. Sikora. On-diagonal estimates on Schrödinger semigroup kernels and reduced heat kernels. Comm. Math. Phys. 188 (1997), no. 1, 233-249.

[22] B. Shiffman and S. Zelditch. Random polynomials of high degree and Levy concentration of measure. Asian J. Math. 7 (2003), no. 4, 627-646.

[23] Z. Rudnick, P. Sarnak. The behavior of eigenstates of arithmetic hyperbolic manifolds. Comm. Math. Phys. 161, No.2, 195-213, (1994).

[24] K. Yajima and G. Zhang. Local smoothing property and Strichartz inequality for Schrödinger equations with potentials superquadratic at infinity. J. Differential Equations (2004), no. 1, 81-110.

[25] S. Zelditch. Uniform distribution of Eigenfunctions on Compact Hyperbolic Surfaces. Duke Math. J., 55, 919-941, (1987).

[26] S. Zelditch. Quantum ergodicity on the sphere Comm. Math. Phys. 146, 61-71 (1992).

[27] S. Zelditch. Random orthonormal bases of spaces of high dimension. Complex patterns in wave functionsdrums, graphs and disorder (P. Trans. Roy. Soc. London A, ed.), vol. 372, S. Gnutzmann and U. Smilansky, Jan. 282014.

[28] S. Zelditch. A random matrix model for quantum mixing. IMRN, No 3, 115-137 (1996)

[29] M. Zworski. Semiclassical analysis. Graduate Studies in Mathematics, Volume 138. AMS (2010).

Didier Robert, Laboratoire de Mathématiques J. Leray, UMR 6629 du CNRS, Université de Nantes, 2, rue de la Houssinière, 44322 Nantes Cedex 03, France • E-mail : didier.robert@univ-nantes.fr

Laurent Thomann, Laboratoire de Mathématiques J. Leray, UMR 6629 du CNRS, Université de Nantes, 2, rue de la Houssinière, 44322 Nantes Cedex 03, France • E-mail : laurent.thomann@univ-nantes.fr 